\title{
The Property of the Solution about Cauchy Problem for Fourth-Order Schrödinger Equation with Critical Time-Oscillating Nonlinearity
}

\author{
Cuihua Guo, ${ }^{1}$ Hongping Ren, ${ }^{2}$ and Shulin Sun ${ }^{3}$ \\ ${ }^{1}$ School of Mathematical Science, Shanxi University, Taiyuan, Shanxi 030006, China \\ ${ }^{2}$ School of Applied Science, Taiyuan University of Science and Technology, Taiyuan 030024, China \\ ${ }^{3}$ School of Mathematics and Computer Science, Shanxi Normal University, Linfen, Shanxi 041004, China \\ Correspondence should be addressed to Hongping Ren; hpren@shu.edu.cn
}

Received 15 April 2014; Accepted 9 June 2014; Published 21 July 2014

Academic Editor: Yushun Wang

Copyright ( 2014 Cuihua Guo et al. This is an open access article distributed under the Creative Commons Attribution License, which permits unrestricted use, distribution, and reproduction in any medium, provided the original work is properly cited.

We study the property of the solution in Sobolev spaces for the Cauchy problem of the following fourth-order Schrödinger equation with critical time-oscillating nonlinearity $i u_{t}+\Delta^{2} u+\theta(\omega t)|u|^{8 /(n-4)} u=0$, where $\omega, t \in R, x \in R^{n}$, and $\theta$ is a periodic function. We obtain the asymptotic property of the solution for the above equation as $|\omega| \rightarrow \infty$ under some conditions.

\section{Introduction}

In this paper we study the Cauchy problem of the following fourth-order Schrödinger equation with a time oscillating critical nonlinearity

$$
\begin{gathered}
i u_{t}+\Delta^{2} u+\theta(\omega t)|u|^{8 /(n-4)} u=0, \quad \text { for } t \in R, x \in R^{n}, \\
u(x, 0)=\varphi(x), \quad x \in R^{n},
\end{gathered}
$$

where $\theta \in C^{1}(R, R)$ is a $\tau$-periodic function and $\omega$ is a real constant. We define the solution of (1) to be $u_{\omega}(x, t)$.

In this paper we plan to study the behavior of $u_{\omega}$ as $|\omega| \rightarrow$ $\infty$. We define $I(\theta)=(1 / \tau) \int_{0}^{\tau} \theta(s) d s$ to be the average value of $\theta(s)$ on $[0, \tau]$. Naturally, we think of the following equation:

$$
\begin{gathered}
i u_{t}+\Delta^{2} u+I(\theta)|u|^{8 /(n-4)} u=0, \quad \text { for } t \in R, x \in R^{n}, \\
u(x, 0)=\varphi(x), \quad x \in R^{n} .
\end{gathered}
$$

We define the solution of (2) to be $u(x, t)$. We will study the relation of $u_{\omega}(x, t)$ and $u(x, t)$ as $|\omega| \rightarrow \infty$.

Definition 1. For two integers $2 \leq q \leq \infty$ and $2 \leq r<\infty$, we say that $(q, r)$ is an admissible pair if the following condition is satisfied:

$$
\frac{4}{q}=n\left(\frac{1}{2}-\frac{1}{r}\right)
$$

To begin with, we give the following local well-posed results by similar techniques in [1].

Proposition 2. Let $\varphi \in H^{2}\left(R^{n}\right)$. Then there exists a unique $H^{2}$-solution $u_{\omega}(x, t)$ on a maximal time interval $\left(-T_{\min }, T_{\max }\right)$ for (1). Moreover, for any admissible pair $(q, r)$ and $I \subset\left(-T_{\min }, T_{\max }\right), u_{\omega}(x, t) \in L^{q}\left(I, H^{2, r}\left(R^{n}\right)\right)$. If $T_{\max }<\infty$ (or $\left.T_{\min }<\infty\right)$, then $\left\|u_{\omega}\right\|_{L^{q}\left(0, T_{\max } ; \dot{H}^{2, r}\left(R^{n}\right)\right)}=$ $\infty\left(\right.$ or $\left.\left\|u_{\omega}\right\|_{L^{q}\left(-T_{\min }, 0 ; \dot{H}^{2, r}\left(R^{n}\right)\right)}=\infty\right)$.

Energy-critical fourth-order Schrödinger equations are very important equations which arise in many physical application fields $[2,3]$. They have been discussed not only by physical researchers but also from mathematical viewpoint by many authors [4-8]. In particular, if the coefficient of nonlinear term is a periodic function in time, condensation and blowup phenomenon may appear from physical experiments. Naturally, from mathematical point, we will analyze why does it happen? What is the condition when condensation or blowup phenomenon appears? What is the property if condensation appears? For the Schrödinger equation, in [9] 
Fang and Han studied the Schrödinger equation with timeoscillating critical nonlinearities. They gave the asymptotic property of local solution and global solution for large frequencies (as $|\omega| \rightarrow \infty$ ). As far as we know, there are few results about the asymptotic property of the solutions for the energy-critical fourth-order Schrödinger equations with periodic coefficient in time. So we will utilize the ideals and techniques of [9] to study the asymptotic behavior of the solution for (1) as $|\omega| \rightarrow \infty$. The difficulty of our work is how to seek power index. The index is dependent to space dimension. And the order of equation is four. This will make it more difficult to look for proper index for us. The aim of this paper is to study the property of the solution for the Cauchy problem of fourth-order Schrödinger equation with time-oscillating critical nonlinearities.

The main results of this work are the following theorems.

Theorem 3. Suppose that $5 \leq n<12$. For arbitrary initial data $\varphi \in H^{2}\left(R^{n}\right)$, we define $u_{\omega}$ and $u$, respectively, as the maximal solution of (1) and (2) on maximal time $\left[0, T_{\max }\right]$. Then we have

$$
\lim _{|\omega| \rightarrow \infty}\left\|u_{\omega}-u\right\|_{L^{q}\left(0, T ; H^{2, r}\left(R^{n}\right)\right)}=0
$$

where $T<T_{\max }$ and $(q, r)$ is arbitrary admissible pair.

Theorem 4. Suppose that $5 \leq n<12$. Let $\gamma=2 n /(n-4), \rho=$ $2 n^{2} /\left(n^{2}-4 n+16\right)$. Assume that $u$ is the global solution of (2), and $u \in L^{\gamma}\left(0, \infty ; L^{\rho}\left(R^{n}\right)\right)$. Then the solution $u_{\omega}$ of $(1)$ is global if $|\omega|$ is sufficiently large. Moreover, we have

$$
\lim _{|\omega| \rightarrow \infty}\left\|u_{\omega}-u\right\|_{L^{q}\left(0, \infty ; H^{2, r}\left(R^{n}\right)\right)}=0
$$

for any admissible pair $(q, r)$.

The succeeding section is devoted to establishing the dispersive estimates for the linear equation related to (1) and (2), and we will present the nonlinear estimates of nonlinearity. In Section 3 we present the proofs of Theorems 3 and 4 .

\section{Notations and Preliminaries}

Given $T>0$ and a function space on $R^{n}$, we denote by $\|\cdot\|_{L^{q}([-T, T] ; X)}$ and $L^{q}([-T, T] ; X)$, respectively, the following norm and the corresponding function space on $[-T, T] \times R^{n}$.

For $1 \leq q<+\infty$,

$$
\|f\|_{L^{q}([-T, T] ; X)}=\left(\int_{-T}^{T}\|f(\cdot, t)\|_{X}^{q} d t\right)^{1 / q} ;
$$

and for $q=\infty$,

$$
\|f\|_{L^{\infty}([-T, T] ; X)}=\text { ess } \sup _{-T<t<T}\|f(\cdot, t)\|_{X} .
$$

Later we will particularly take $X=H^{s, r}\left(R^{n}\right)(s \in R, 1 \leq r \leq$ $\infty)$. For simplicity of the notations, we, respectively, abbreviate $\|\cdot\|_{L^{q}\left([-T, T] ; L^{r}\left(R^{n}\right)\right)}$ and $\|\cdot\|_{L^{q}\left([-T, T] ; H^{s, r}\left(R^{n}\right)\right)}$ as, respectively, $\|\cdot\|_{L_{T}^{q} L_{x}^{r}}$ and $\|\cdot\|_{L_{T}^{q} H_{x}^{s, r}}$. In particular, for the case $q=r$, we abbreviate $\|\cdot\|_{L^{q}\left([-T, T] ; L^{r}\left(R^{n}\right)\right)}$ as $\|\cdot\|_{L_{T, x}^{q}}$. We also abbreviate $H^{s, 2}\left(R^{n}\right)=H^{s}\left(R^{n}\right)$. In the following, we will introduce our four working spaces.

For any time interval $T$, we denote

$$
\begin{gathered}
\|u\|_{X^{0}(T)}=\|u\|_{L_{T}^{n(n+4) / 2(n-4)} L_{x}^{2 n^{2}(n+4) /(n-4)\left(n^{2}+4 n-16\right)},} \\
\|u\|_{X(T)}=\left\||\nabla|^{8 /(n+4)} u\right\|_{L_{T}^{n(n+4) / 2(n-4)} L_{x}^{2 n^{2}(n+4) /\left(n^{3}-16 n+64\right)},} \\
\|u\|_{Y(T)}=\left\||\nabla|^{8 /(n+4)} u\right\|_{L_{T}^{n / 2} L_{x}^{2 n^{2}(n+4) /\left(n^{3}+8 n^{2}+16 n-64\right)},}, \\
\|u\|_{S^{0}(T)}=\sup _{(q, r) \text { is admissible }}\|u\|_{L_{T}^{n / 2} L_{x}^{q} r^{2}}
\end{gathered}
$$

The fundamental solution of the linear equation related to (1) and (2) is given by the following oscillatory integral:

$$
I(x, t)=(2 \pi)^{-n} \int_{R^{n}} e^{i x \cdot \xi+i t|\xi|^{4}} d \xi .
$$

We denote by $W(t)(t \in R)$ the fundamental solution operator

$$
W(t) \varphi=I(x, t) * \varphi(x), \quad \varphi(x) \in S^{\prime}\left(R^{n}\right) .
$$

So (1) and (2) have the following integral forms, respectively:

$$
\begin{aligned}
& u_{\omega}(x, t) \\
& =W(t) \varphi+i \int_{0}^{t} W(t-s) \theta(\omega s)\left|u_{\omega}(s)\right|^{8 /(n-4)} u_{\omega}(s) d s \\
& u(x, t)=W(t) \varphi+i I(\theta) \int_{0}^{t} W(t-s)|u(s)|^{8 /(n-4)} u(s) d s
\end{aligned}
$$

Lemma 5 ((Strichartz estimates) (see [10])). Assume $s \geq$ $0, \varphi(x) \in H^{s}\left(R^{n}\right), f(x, t) \in L_{T}^{\gamma^{\prime}} L_{x}^{\rho^{\prime}}$, and $u(x, t)$ is a solution on $[0, T]$ of the following initial value problem:

$$
\begin{gathered}
i u_{t}+\Delta^{2} u+f(x, t)=0, \quad \text { for } t \in R, x \in R^{n}, \\
u(x, 0)=\varphi(x), \quad x \in R^{n},
\end{gathered}
$$

then for all admissible pairs $(q, r)$ and $(\gamma, \rho)$, we have

$$
\begin{gathered}
\|W(t) \varphi\|_{L_{T}^{q} H_{x}^{s, r}} \leq C\|\varphi\|_{H^{s}\left(R^{n}\right)}, \\
\left\|\int_{0}^{t} W(t-s) f(\cdot, s) d s\right\|_{L_{T}^{q} L_{x}^{r}} \leq C\|f\|_{L_{T}^{\gamma^{\prime}} L_{x}^{\rho^{\prime}}}
\end{gathered}
$$

Lemma 6. Let $T$ be a compact time interval containing $t_{0}$. Then we have

$$
\left\|\int_{t_{0}}^{t} W(t-s) F(\cdot, s) d s\right\|_{X(T)} \leq C\|F\|_{Y(T)} .
$$

Proof. By the definition of $W(t)$, we obtain

$$
\begin{aligned}
& \|W(t-s) F(\cdot, s)\|_{L_{x}^{2 n^{2}(n+4) /\left(n^{3}-16 n+64\right)}} \\
& \leq C|t-s|^{-n^{3}+4 n-16 / n(n+4)}\|F(x, t)\|_{L_{x}^{2 n^{2}(n+4) /\left(n^{3}+8 n^{2}+16 n-64\right)}} .
\end{aligned}
$$


Using Hardy-Littlewood-Sobolev inequality, we have

$$
\begin{aligned}
& \left\|\int_{t_{0}}^{t} W(t-s) F(\cdot, s) d s\right\|_{L_{T}^{n(n+4) / 2(n-4)} L_{x}^{2 n^{2}(n+4) /\left(n^{3}-16 n+64\right)}} \\
& \leq C\|F(x, t)\|_{L_{T}^{n / 2} L_{x}^{2 n^{2}(n+4) /\left(n^{3}+8 n^{2}+16 n-64\right)}}
\end{aligned}
$$

which completes the proof.

Lemma 7. For any compact time interval $T$, we have

$$
\begin{gathered}
\|u\|_{X^{0}(T)} \lesssim\|u\|_{X(T)} \leqslant\left\|D^{2} u\right\|_{S^{0}(T)}, \\
\|u\|_{X(T)} \lesssim\|u\|_{L_{T, x}^{2(n+4) /(n-4)}}^{1 /(n+4)}\left\|D^{2} u\right\|_{S^{0}(T)}^{(n+3) /(n+4)} .
\end{gathered}
$$

Proof. Using Sobolev embedding (see [11]) $\dot{H}^{8 /(n+4), 2 n^{2}(n+4) /\left(n^{3}-16 n+64\right)}\left(R^{n}\right) \hookrightarrow L^{2 n^{2}(n+4) /(n-4)\left(n^{2}+4 n-16\right)}\left(R^{n}\right)$, we have

$$
\|u\|_{X^{0}(T)} \leq C\|u\|_{X(T)} .
$$

Similarly, using Sobolev embedding $\dot{H}^{2,2 n^{2}(n+4) /\left(n^{3}+4 n^{2}-16 n+64\right)}$ $\left(R^{n}\right) \hookrightarrow \dot{H}^{8 /(n+4), 2 n^{2}(n+4) /\left(n^{3}-16 n+64\right)}\left(R^{n}\right)$ and noting that $(n(n+$ 4) $\left./ 2(n-4), 2 n^{2}(n+4) /\left(n^{3}+4 n^{2}-16 n+64\right)\right)$ is an admissible pair, we have

$$
\|u\|_{X(T)} \leq C\left\|D^{2} u\right\|_{L_{t}^{n(n+4) / 2(n-4)} L_{x}^{22^{2}(n+4) /\left(n^{3}+4 n^{2}-16 n+64\right)}} \leq C\left\|D^{2} u\right\|_{S^{0}(T)},
$$

which completes the proof of the first inequality.

Next we prove the second inequality.

Using interpolation inequality (see [12]), we obtain

$$
\begin{aligned}
& \left\||\nabla|^{8 /(n+4)} u\right\|_{L_{T}^{n(n+4) / 2(n+4)} L_{x}^{2 n^{2}(n+4) /\left(n^{3}-16 n+64\right)}} \\
& \leq C\|u\|_{L_{T, x}^{2(n+4) /(n-4)}}^{1 /(n+4)} \\
& \quad \times\left\||\nabla|^{8 /(n+3)} u\right\|_{L_{T}^{2 n(n+3)(n+4) /(n-4)(3 n+16)} L_{x}^{2 n^{2}(n+3)(n+4) /\left(n^{4}+3 n^{3}-12 n^{2}+256\right)}}^{(n+3) /(n+4)} .
\end{aligned}
$$

Using Sobolev embedding, we obtain

$$
\begin{aligned}
& \left\||\nabla|^{8 /(n+3)} u\right\|_{L_{T}^{2 n(n+3)(n+4) /(n-4)(3 n+16)} L_{x}^{2 n^{2}(n+3)(n+4) /\left(n^{4}+3 n^{3}-12 n^{2}+256\right)}} \\
& \leq C\left\|D^{2} u\right\|_{L_{T}^{2 n(n+3)(n+4) /(n-4)(3 n+16)} L_{x}^{2 n^{2}(n+3)(n+4) /\left(n^{4}+7 n^{3}-16 n+256\right)} .} .
\end{aligned}
$$

So we have

$$
\begin{aligned}
& \left\||\nabla|^{8 /(n+4)} u\right\|_{L_{T}^{n(n+4) / 2(n+4)} L_{x}^{22^{2}(n+4) /\left(n^{3}-16 n+64\right)}} \\
& \leq C\|u\|_{L_{T, x}^{2(n+4) /(n-4)}}^{1 /(n+4)}\left\|D^{2} u\right\|_{S^{0}(T)}^{(n+3) /(n+4)}
\end{aligned}
$$

which completes the proof of the second inequality.

Assume that the nonlinear function $F(u)=|u|^{8 /(n-4)} u$. Then we have the following two lemmas.
Lemma 8. Let $T$ be a compact time interval. Then, we have

$$
\begin{gathered}
\|F(u)\|_{Y(T)} \leq C\|u\|_{X(T)}^{(n+4) /(n-4)} \\
\left\|F_{z}(u+v) w\right\|_{Y(T)}+\left\|F_{\bar{z}}(u+v) \bar{w}\right\|_{Y(T)} \\
\leq C\left(\|u\|_{X(T)}^{4(n+8) /\left(n^{2}-16\right)\left\|D^{2} u\right\|_{S^{0}(T)}^{4 n /\left(n^{2}-16\right)}}\right. \\
\left.+\|v\|_{X(T)}^{4(n+8) /\left(n^{2}-16\right)}\left\|D^{2} v\right\|_{S^{0}(T)}^{4 n /\left(n^{2}-16\right)}\right)\|w\|_{X(T)} .
\end{gathered}
$$

Proof. Using Lemma A.11 of [13] and Lemma 7, we have

$$
\begin{aligned}
& \|F(u)\|_{Y(T)} \\
& \leq C\left\||u|^{8 /(n-4)}\right\|_{L_{T}^{n(n+4) / 16} L_{x}^{n^{2}(n+4) / 4\left(n^{2}+4 n-16\right)}} \\
& \quad \times\left\||\nabla|^{8 /(n+4)} u\right\|_{L_{T}^{n(n+4) / 2(n-4)} L_{x}^{2 n^{2}(n+4) /\left(n^{3}-16 n+64\right)}} \\
& =C\|u\|_{L_{T}^{n /(n+4) / 2(n-4)} L_{x}^{2 n^{2}(n+4) /(n-4)\left(n^{2}+4 n-16\right)}}^{8(n-4)} \\
& \quad \times\left\||\nabla|^{8 /(n+4)} u\right\|_{L_{T}^{n(n+4) / 2(n-4)} L_{x}^{2 n^{2}(n+4) /\left(n^{3}-16 n+64\right)}} \\
& =\|u\|_{X^{0}(T)}^{8 /(n-4)}\|u\|_{X(T)} \leq C\|u\|_{X(T)}^{(n+4) /(n-4)} .
\end{aligned}
$$

For the second inequality, we have

$$
\begin{aligned}
& \left\|F_{z}(u+v) w\right\|_{Y(T)} \\
& =\left\||\nabla|^{8 /(n+4)}\left(F_{z}(u+v) w\right)\right\|_{L_{T}^{n / 2} L_{x}^{22^{2}(n+4) /\left(n^{3}+8 n^{2}+16 n-64\right)}} \\
& \leq C\left(\left\|F_{z}(u+v)\right\|_{L_{T}^{n(n+4) / 16} L_{x}^{n^{2}(n+4) /\left(4 n^{2}+16 n-64\right)}}\right. \\
& \times\left\||\nabla|^{8 /(n+4)} w\right\|_{L_{T}^{n(n+4) / 2(n-4)} L_{x}^{2 n^{2}(n+4) /\left(n^{3}-16 n+64\right)}} \\
& +\left\||\nabla|^{8 /(n+4)} F_{z}(u+v)\right\|_{L_{T}^{n(n+4) / 16} L_{x}^{n^{2}(n+4) / 4\left(n^{2}+6 n-16\right)}} \\
& \left.\times\|w\|_{L_{T}^{n(n+4) / 2(n-4)} L_{x}^{2 n^{2}(n+4) /(n-4)\left(n^{2}+4 n-16\right)}}\right) \\
& \leq C\left(\|u+v\|_{L_{T}^{n(n+4) / 2(n-4)} L_{x}^{2 n^{2}(n+4) /(n-4)\left(n^{2}+4 n-16\right)}}^{8 /(n-4)}\right. \\
& \times\left\||\nabla|^{8 /(n+4)} w\right\|_{L_{T}^{n(n+4) / 2(n-4)} L_{x}^{2 n^{2}(n+4) /\left(n^{3}-16 n+64\right)}} \\
& +\left\||\nabla|^{8 /(n+4)} F_{z}(u+v)\right\|_{L_{T}^{n(n+4) / 16} L_{x}^{n^{2}(n+4) / 4\left(n^{2}+6 n-16\right)}} \\
& \left.\times\|w\|_{L_{T}^{n(n+4) / 2(n-4)} L_{x}^{22^{2}(n+4) /(n-4)\left(n^{2}+4 n-16\right)}}\right) \\
& \leq C\left(\|u+v\|_{X^{0}(T)}^{8 /(n-4)}\|w\|_{X(T)}\right.
\end{aligned}
$$

$$
+\left\||\nabla|^{8 /(n+4)} F_{z}(u+v)\right\|_{\left.L_{T}^{n(n+4) / 16} L_{x}^{n^{2}(n+4) / 4\left(n^{2}+6 n-16\right)}\|w\|_{X^{0}(T)}\right)}
$$




$$
\begin{aligned}
\leq C( & \|u+v\|_{X^{0}(T)}^{8 /(n-4)}\|w\|_{X(T)} \\
& +\left\||\nabla|^{8 /(n+4)} F_{z}(u+v)\right\|_{\left.L_{T}^{n(n+4) / 16} L_{x}^{n^{2}(n+4) / 4\left(n^{2}+6 n-16\right)}\|w\|_{X(T)}\right) .}
\end{aligned}
$$

For $5 \leq n<12$, using Lemma A.11 of [13] and Lemma 7, we obtain

$$
\begin{aligned}
& \left\||\nabla|^{8 /(n+4)} F_{z}(u+v)\right\|_{L_{T}^{n(n+4) / 16} L_{x}^{n^{2}(n+4) /\left(4 n^{2}+24 n-64\right)}} \\
& \leq C\left\||u+v|^{(12-n) /(n-4)}\right\|_{L_{T}^{n(n+4) / 2(12-n)} L_{x}^{2 n^{2}(n+4) /(12-n)\left(n^{2}+4 n-16\right)}} \\
& \quad \times\left\||\nabla|^{8 /(n+4)}(u+v)\right\|_{L_{T}^{n(n+4) / 2(n-4)} L_{x}^{2 n^{2}(n+4) /\left(n^{3}-16 n+64\right)}} \\
& \leq C\|u+v\|_{L_{T}^{n(n+4) / 2(n-4)} L_{x}^{2 n^{2}(n+4) /(n-4)\left(n^{2}+4 n-16\right)}}^{(12-n) /(n-4)} \\
& \quad \times\left\||\nabla|^{8 /(n+4)}(u+v)\right\|_{L_{T}^{n(n+4) / 2(n-4)} L_{x}^{2 n^{2}(n+4) /\left(n^{3}-16 n+64\right)}} \\
& \leq C\|u+v\|_{X^{0}(T)}^{(12-n) /(n-4)}\|u+v\|_{X(T)} \\
& \leq C\|u+v\|_{X(T)}^{8 /(n-4)} \leq C\left(\|u\|_{X(T)}^{8 /(n-4)}+\|v\|_{X(T)}^{8 /(n-4)}\right) \\
& \leq C\left(\|u\|_{X(T)}^{4(n+8) /\left(n^{2}-16\right)}\left\|D^{2} u\right\|_{S^{0}(T)}^{4 n /\left(n^{2}-16\right)}\right. \\
& \left.\quad+\|v\|_{X(T)}^{4(n+8) /\left(n^{2}-16\right)}\left\|D^{2} v\right\|_{S^{0}(T)}^{4 n /\left(n^{2}-16\right)}\right)
\end{aligned}
$$

For $n \geq 12$, using Lemma A.12 of [13] $(\alpha=8 /(n-4), s=$ $8 /(n+4), \sigma=n /(n+4))$ and Sobolev inequality, we have

$$
\begin{aligned}
& \left\||\nabla|^{8 /(n+4)} F_{z}(u+v)\right\|_{L_{x}^{n^{2}(n+4) /\left(4 n^{2}+24 n-64\right)}} \\
& \leq C\left\||u+v|^{32 / n(n-4)}\right\|_{L_{x}^{2 n^{2}(n+4) /(n-4)\left(n^{2}+4 n-16\right)}} \\
& \quad \times\left\||\nabla|^{n /(n+4)}(u+v)\right\|_{L_{x}^{2 n^{2}(n+4) /\left(n^{3}+2 n^{2}-32 n+64\right)}}^{8 / n} \\
& \leq C\left\||\nabla|^{n /(n+4)}(u+v)\right\|_{L_{x}^{2 n^{2}(n+4) /\left(n^{3}+2 n^{2}-32 n+64\right)}}^{8 /(n-4)} .
\end{aligned}
$$

From (28), using interpolation inequality and Lemma 7, we have

$$
\begin{aligned}
& \left\||\nabla|^{8 /(n+4)} F_{z}(u+v)\right\|_{L_{T}^{n(n+4) / 16} L_{x}^{n^{2}(n+4) / 4\left(n^{2}+6 n-16\right)}} \\
& \leq C\left\||\nabla|^{n /(n+4)}(u+v)\right\|_{L_{T}^{n(n+4) / 2(n-4)} L_{x}^{n^{2}(n+4) /\left(n^{3}+2 n^{2}-32 n+64\right)}}^{8 /(n-4)} \\
& \leq C\left(\left\||\nabla|^{n /(n+4)} u\right\|_{L_{T}^{n(n+4) / 2(n-4)} L_{x}^{n^{2}(n+4) /\left(n^{3}+2 n^{2}-32 n+64\right)}}^{8 /(n-4)}\right. \\
& \left.+\left\||\nabla|^{n /(n+4)} v\right\|_{L_{T}^{n(n+4) / 2(n-4)} L_{x}^{n^{2}(n+4) /\left(n^{3}+2 n^{2}-32 n+64\right)}}^{8 /(n-4)}\right) \\
& \leq C\left(\|u\|_{L_{T}^{n(n+4) / 2(n-4)} L_{x}^{2 n^{2}(n+4) /(n-4)\left(n^{2}+4 n-16\right)}}^{4(n+8) /\left(n^{2}-16\right)}\right. \\
& \times\left\|D^{2} u\right\|_{L_{T}^{n(n+4) / 2(n-4)} L_{x}^{2 n^{2}(n+4) /\left(n^{3}+4 n^{2}-16 n+64\right)}}^{4 n /\left(n^{2}-16\right)} \\
& +\|v\|_{L_{T}^{n(n+4) / 2(n-4)} L_{x}^{2 n^{2}(n+4) /(n-4)\left(n^{2}+4 n-16\right)}}^{4(n+8) /\left(n^{2}-16\right)}
\end{aligned}
$$

$$
\begin{gathered}
\left.\times\left\|D^{2} v\right\|_{L_{T}^{n(n+4) / 2(n-4)} L_{x}^{2 n^{2}(n+4) /\left(n^{3}+4 n^{2}-16 n+64\right)}}^{4 n /\left(n^{2}-16\right)}\right) \\
\leq C\left(\|u\|_{X^{0}(T)}^{4(n+8) /\left(n^{2}-16\right)}\left\|D^{2} u\right\|_{S^{0}(T)}^{4 n /\left(n^{2}-16\right)}\right. \\
\left.+\|v\|_{X^{0}(T)}^{4(n+8) /\left(n^{2}-16\right)}\left\|D^{2} v\right\|_{S^{0}(T)}^{4 n /\left(n^{2}-16\right)}\right) \\
\leq C\left(\|u\|_{X(T)}^{4(n+8) /\left(n^{2}-16\right)}\left\|D^{2} u\right\|_{S^{0}(T)}^{4 n /\left(n^{2}-16\right)}\right. \\
\left.+\|v\|_{X(T)}^{4(n+8) /\left(n^{2}-16\right)}\left\|D^{2} v\right\|_{S^{0}(T)}^{4 n /\left(n^{2}-16\right)}\right) .
\end{gathered}
$$

From (26), (27), and (29), we have

$$
\begin{aligned}
& \left\|F_{z}(u+v) w\right\|_{Y(T)} \\
& \leq C\left(\|u\|_{X(T)}^{4(n+8) /\left(n^{2}-16\right)}\left\|D^{2} u\right\|_{S^{0}(T)}^{4 n /\left(n^{2}-16\right)}\right. \\
& \left.\quad+\|v\|_{X(T)}^{4(n+8) /\left(n^{2}-16\right)}\left\|D^{2} v\right\|_{S^{0}(T)}^{4 n /\left(n^{2}-16\right)}\right) .
\end{aligned}
$$

Similarly, we can prove

$$
\begin{aligned}
& \left\|F_{\bar{z}}(u+v) \bar{w}\right\|_{Y(T)} \\
& \leq C\left(\|u\|_{X(T)}^{4(n+8) /\left(n^{2}-16\right)}\left\|D^{2} u\right\|_{S^{0}(T)}^{4 n /\left(n^{2}-16\right)}\right. \\
& \left.\quad+\|v\|_{X(T)}^{4(n+8) /\left(n^{2}-16\right)}\left\|D^{2} v\right\|_{S^{0}(T)}^{4 n /\left(n^{2}-16\right)}\right),
\end{aligned}
$$

which completes the proof.

Using Lemma 8, we immediately obtain the following lemma.

Lemma 9. Let $n \geq 5$. Then we have

$$
\begin{aligned}
& \|F(u)-F(v)\|_{Y(T)} \\
& \leq C\left(\|v\|_{X(T)}^{4(n+8) /\left(n^{2}-16\right)}\left\|D^{2} v\right\|_{S^{0}(T)}^{4 n /\left(n^{2}-16\right)}+\|u-v\|_{X(T)}^{4(n+8) /\left(n^{2}-16\right)}\right. \\
& \left.\quad \times\left\|D^{2}(u-v)\right\|_{S^{0}(T)}^{4 n /\left(n^{2}-16\right)}\right)\|u-v\|_{X(T)} .
\end{aligned}
$$

Using the proof techniques in [14], similarly we can obtain the following lemma.

Lemma 10. For any admissible pair $(\gamma, \rho),(q, r), f \in L_{T}^{\gamma^{\prime}} L_{x}^{\rho^{\prime}}$, we have

$$
\begin{aligned}
& \| \int_{t_{0}}^{t} \theta(\omega s) W(t-s) f(\cdot, s) d s \\
& \quad-I(\theta) \int_{t_{0}}^{t} W(t-s) f(\cdot, s) d s \|_{L_{T}^{q} L_{x}^{r}} \longrightarrow 0 \quad \text { as }|\omega| \longrightarrow \infty .
\end{aligned}
$$


Lemma 11. Let $\gamma=2 n /(n-4), \rho=2 n^{2} /\left(n^{2}-4 n+16\right)$. For any initial value $\varphi \in H^{2}\left(R^{n}\right), 5 \leq n<12$, assume that

$$
\lim _{|w| \rightarrow \infty} \sup \left\|D^{2} u_{\omega}\right\|_{L^{\gamma}\left(0, l ; L^{\rho}\left(R^{n}\right)\right)}<+\infty,
$$

then we have

$$
\left\|u_{\omega}-u\right\|_{L^{q}\left(0, l ; H^{2, r}\left(R^{n}\right)\right)} \longrightarrow 0 \text { as }|\omega| \longrightarrow \infty,
$$

where $(q, r)$ is admissible pair.

Proof. (1) First we prove that $\left\|u_{\omega}(x, t)-u(x, t)\right\|_{L^{q}\left(0, l ; L^{r}\right)} \rightarrow$ 0 as $|\omega| \rightarrow \infty$.

By (34), there must be constants $L$ and $M$ such that

$$
\sup _{|\omega| \geq L}\left\|D^{2} u_{\omega}\right\|_{L^{\gamma}\left(0, l ; L^{\rho}\left(R^{n}\right)\right)} \leq M
$$

From (11) and (12), we have

$$
u_{\omega}(x, t)-u(x, t)=i\left(I_{1}+I_{2}\right),
$$

where $I_{1}=\int_{0}^{t} \theta(\omega s) W(t-s)\left[\left|u_{\omega}(s)\right|^{8 /(n-4)} u_{\omega}(s)-|u(s)|^{8 /(n-4)}\right.$ $u(s)] d s$ and $I_{2}=\int_{0}^{t}[\theta(\omega s)-I(\theta)] W(t-s)|u(s)|^{8 /(n-4)} u(s) d s$.

By Lemma 10, we have

$$
\left\|I_{2}\right\|_{L^{q}\left(0, l ; L^{r}\left(R^{n}\right)\right)}:=C \varepsilon_{\omega} \longrightarrow 0 \text { as }|\omega| \longrightarrow \infty .
$$

Using Hölder inequality and Sobolev embedding inequality, we obtain

$$
\begin{aligned}
& \left\|I_{1}\right\|_{L^{q}\left(0, l ; L^{r}\left(R^{n}\right)\right)} \\
& \leq C\left\|\left|u_{\omega}(s)\right|^{8 /(n-4)} u_{\omega}(s)-|u(s)|^{8 /(n-4)} u(s)\right\|_{L^{\gamma^{\prime}}\left(0, l ; L^{L^{\prime}}\left(R^{n}\right)\right)} \\
& \leq C\left(\left\|D^{2} u_{\omega}\right\|_{L^{\gamma}\left(0, l ; L^{\rho}\left(R^{n}\right)\right)}^{8 /(n-4)}+\left\|D^{2} u\right\|_{L^{\gamma}\left(0, l ; L^{\rho}\left(R^{n}\right)\right)}^{8 /(n-4)}\right) \\
& \quad \times\left\|u_{\omega}-u\right\|_{L^{\gamma}\left(0, l ; L^{\rho}\left(R^{n}\right)\right)} \\
& \leq C\left(M^{8 /(n-4)}+N^{8 /(n-4)}\right)\left\|u_{\omega}-u\right\|_{L^{\gamma}\left(0, l ; L^{\rho}\left(R^{n}\right)\right)},
\end{aligned}
$$

where $N=\|u\|_{L^{\gamma}\left(0, l ; H^{2, \rho}\left(R^{n}\right)\right)}$.

Using (38) and (39), we have

$$
\begin{aligned}
& \left\|u_{\omega}(x, t)-u(x, t)\right\|_{L^{q}\left(0, l ; L^{r}\left(R^{n}\right)\right)}=\left\|I_{1}+I_{2}\right\|_{L^{q}\left(0, l ; L^{r}\left(R^{n}\right)\right)} \\
& \leq\left\|I_{1}\right\|_{L^{q}\left(0, l ; L^{r}\left(R^{n}\right)\right)}+\left\|I_{2}\right\|_{L^{q}\left(0, l ; L^{r}\left(R^{n}\right)\right)} \\
& \leq C \varepsilon_{\omega}+C\left(M^{8 /(n-4)}+N^{8 /(n-4)}\right) \\
& \quad \times\left\|u_{\omega}(x, t)-u(x, t)\right\|_{L^{\gamma}\left(0, l ; L^{\rho}\left(R^{n}\right)\right)} .
\end{aligned}
$$

In the following we will prove that $\| u_{\omega}(x, t)-$ $u(x, t) \|_{L^{\gamma}\left(0, l ; L^{\rho}\left(R^{n}\right)\right)}$.

Since $\sup _{|\omega| \geq L}\left\|D^{2} u_{\omega}\right\|_{L^{\gamma}\left(0, l ; L^{\rho}\left(R^{n}\right)\right)} \leq M$, we can divide the time interval $[0, l]$ into subintervals $\left[t_{i}, t_{i+1}\right], i=0, \ldots, J-$ 1 , where $t_{0}=0, t_{J-1}=l$, such that in each part $C\left(\left\|D^{2} u_{\omega}\right\|_{L^{\gamma}\left(t_{i}, t_{i+1} ; L^{\rho}\left(R^{n}\right)\right)}^{8 /(n-4)}+\left\|D^{2} u\right\|_{L^{\gamma}\left(t_{i}, t_{i+1} ; L^{\rho}\left(R^{n}\right)\right)}^{8 /(n-4)}\right)=1 / 2$.
On $\left[t_{0}, t_{1}\right]$, since $u_{\omega}\left(t_{0}\right)=u\left(t_{0}\right)=\varphi$, we have

$$
\begin{gathered}
\left\|u_{\omega}(x, t)-u(x, t)\right\|_{L^{q}\left(t_{0}, t_{1} ; L^{r}\left(R^{n}\right)\right)} \\
\leq C \varepsilon_{\omega}+C\left(\left\|D^{2} u_{\omega}\right\|_{L^{\gamma}\left(t_{0}, t_{1} ; L^{\rho}\left(R^{n}\right)\right)}^{8 /(n-4)}\right. \\
\left.+\left\|D^{2} u\right\|_{L^{\gamma}\left(t_{0}, t_{1} ; L^{\rho}\left(R^{n}\right)\right)}^{8 /(n-4)}\right) \\
\times\left\|u_{\omega}(x, t)-u(x, t)\right\|_{L^{\gamma}\left(t_{0}, t_{1} ; L^{\rho}\left(R^{n}\right)\right)} \\
\leq C \varepsilon_{\omega}+\frac{1}{2}\left\|u_{\omega}(x, t)-u(x, t)\right\|_{L^{\gamma}\left(t_{0}, t_{1} ; L^{\rho}\left(R^{n}\right)\right)} .
\end{gathered}
$$

For the case $(q, r)=(\gamma, \rho)$, we have

$$
\left\|u_{\omega}(x, t)-u(x, t)\right\|_{L^{\gamma}\left(t_{0}, t_{1} ; L^{\rho}\left(R^{n}\right)\right)} \leq 2 C \varepsilon_{\omega} .
$$

For the case $(q, r)=(\infty, 2)$, we have

$$
\left\|u_{\omega}(x, t)-u(x, t)\right\|_{L^{\infty}\left(t_{0}, t_{1} ; L^{2}\left(R^{n}\right)\right)} \leq 2 C \varepsilon_{\omega} .
$$

On $\left[t_{1}, t_{2}\right]$, we have

$$
\begin{aligned}
& \left\|u_{\omega}(x, t)-u(x, t)\right\|_{L^{q}\left(t_{1}, t_{2} ; L^{r}\left(R^{n}\right)\right)} \\
& \leq\left\|u_{\omega}\left(t_{1}\right)-u\left(t_{1}\right)\right\|_{L^{2}\left(R^{n}\right)}+C \varepsilon_{\omega} \\
& \quad+\frac{1}{2}\left\|u_{\omega}(x, t)-u(x, t)\right\|_{L^{\gamma}\left(t_{1}, t_{2} ; L^{\rho}\left(R^{n}\right)\right)} \\
& \leq 3 C \varepsilon_{\omega}+\frac{1}{2}\left\|u_{\omega}(x, t)-u(x, t)\right\|_{L^{\gamma}\left(t_{1}, t_{2} ; L^{\rho}\left(R^{n}\right)\right)} .
\end{aligned}
$$

Similarly for the case $(q, r)=(\gamma, \rho)$, we have

$$
\left\|u_{\omega}(x, t)-u(x, t)\right\|_{L^{\gamma}\left(t_{1}, t_{2} ; L^{\rho}\left(R^{n}\right)\right)} \leq 6 C \varepsilon_{\omega} .
$$

For the case $(q, r)=(\infty, 2)$, we have

$$
\left\|u_{\omega}(x, t)-u(x, t)\right\|_{L^{\infty}\left(t_{1}, t_{2} ; L^{2}\left(R^{n}\right)\right)} \leq 6 C \varepsilon_{\omega} .
$$

By induction, we have

$$
\begin{gathered}
\left\|u_{\omega}(x, t)-u(x, t)\right\|_{L^{\gamma}\left(t_{i}, t_{i+1} ; L^{\rho}\left(R^{n}\right)\right)} \leq 2\left(2^{i+1}-1\right) C \varepsilon_{\omega}, \\
\left\|u_{\omega}(x, t)-u(x, t)\right\|_{L^{\infty}\left(t_{i}, t_{i+1} ; L^{2}\left(R^{n}\right)\right)} \leq 2\left(2^{i+1}-1\right) C \varepsilon_{\omega},
\end{gathered}
$$

for $i=0, \ldots, J-1$.

So we have

$$
\begin{aligned}
& \left\|u_{\omega}(x, t)-u(x, t)\right\|_{L^{\gamma}\left(0, l ; L^{\rho}\left(R^{n}\right)\right)} \\
& \leq \sum_{i=0}^{J-1} 2\left(2^{i+1}-1\right) C \varepsilon_{\omega} \\
& =\left[4\left(2^{J}-1\right)-2 J\right] C \varepsilon_{\omega} \longrightarrow 0 \text { as }|\omega| \longrightarrow \infty .
\end{aligned}
$$

Using the above estimate and (40), we have $\left\|u_{\omega}(x, t)-u(x, t)\right\|_{L^{q}\left(0, l ; L^{r}\left(R^{n}\right)\right)}$

$$
\begin{aligned}
\leq & C \varepsilon_{\omega}+C\left(M^{8 /(n-4)}+N^{8 /(n-4)}\right) \\
& \times\left\|u_{\omega}(x, t)-u(x, t)\right\|_{L^{\gamma}\left(0, l ; L^{\rho}\left(R^{n}\right)\right)} \longrightarrow 0 \text { as }|\omega| \longrightarrow \infty .
\end{aligned}
$$


(2) In the following we discuss the estimate $\| \nabla\left(u_{\omega}-\right.$ u) $\|_{L^{q}\left(0, l ; L^{r}\left(R^{n}\right)\right)}$.

By (11) and (12), we have

$$
\nabla\left(u_{\omega}-u\right)=i\left(J_{1}+J_{2}\right)
$$

where $J_{1}=\int_{0}^{t} \theta(\omega s) W(t-s) \nabla\left(\left|u_{\omega}\right|^{8 /(n-4)} u_{\omega}-|u|^{8 /(n-4)} u\right) d s$ and $J_{2}=\int_{0}^{t}[\theta(\omega s)-I(\theta)] W(t-s) \nabla\left(|u|^{8 /(n-4)} u\right) d s$.

Using (11)-(12) and Lemma 5, we obtain

$$
\begin{aligned}
& \left\|\nabla\left(u_{\omega}-u\right)\right\|_{S^{0}\left(0, t_{1}\right)} \\
& \leq C\left\|\nabla\left(u_{\omega}(0)-u(0)\right)\right\|_{L^{2}\left(R^{n}\right)} \\
& +C\left(\left\|J_{1}\right\|_{L^{q}\left(0, t_{1} ; L^{r}\left(R^{n}\right)\right)}+\left\|J_{2}\right\|_{L^{q}\left(0, t_{1} ; L^{r}\left(R^{n}\right)\right)}\right) \\
& \leq C\left\|\nabla\left(u_{\omega}(0)-u(0)\right)\right\|_{L^{2}\left(R^{n}\right)} \\
& +C \| \nabla\left(\left|u_{\omega}\right|^{8 /(n-4)} u_{\omega}\right. \\
& \left.\quad-|u|^{8 /(n-4)} u\right) \|_{L^{2 n(n+4) /\left(n^{2}+4 n+8\right)}\left(0, t_{1} ; L^{2 n^{2}(n+4) /\left(n^{3}+8 n^{2}+16 n-32\right)}\left(R^{n}\right)\right)}
\end{aligned}
$$

$+C\left\|J_{2}\right\|_{L^{q}\left(0, t_{1} ; L^{r}\left(R^{n}\right)\right)}$.

$$
\begin{aligned}
& \text { Noting that } \\
& \left\|\nabla\left(|u|^{8 /(n-4)} u\right)\right\|_{L^{\gamma^{\prime}}\left(0, t_{1} ; L^{\rho^{\prime}}\left(R^{n}\right)\right)} \\
& \leq\left\||u|^{8 /(n-4)}\right\|_{L^{n / 4}\left(0, t_{1} ; L^{n^{2} / 4(n-4)}\left(R^{n}\right)\right)}\|\nabla u\|_{L^{\gamma}\left(0, t_{1} ; L^{\rho}\left(R^{n}\right)\right)} \\
& =\|u\|_{L^{2 n /(n-4)}\left(0, t_{1} ; L^{2 n^{2} /(n-4)^{2}}\left(R^{n}\right)\right)}^{8 /(n-4)}\|\nabla u\|_{L^{\gamma}\left(0, t_{1} ; L^{\rho}\left(R^{n}\right)\right)} \\
& \leq C\left\|D^{2} u\right\|_{L^{\gamma}\left(0, t_{1} ; L^{\rho}\left(R^{n}\right)\right)}^{8 /(n-4)}\|\nabla u\|_{L^{\gamma}\left(0, t_{1} ; L^{\rho}\left(R^{n}\right)\right)},
\end{aligned}
$$

so $\nabla\left(|u|^{8 /(n-4)} u\right) \in L^{\gamma^{\prime}}\left(0, t_{1} ; L^{\rho^{\prime}}\left(R^{n}\right)\right)$.

Using Lemma 5 and Lemma 10, we have

$$
\left\|J_{2}\right\|_{L^{q}\left(0, t_{1} ; L^{r}\left(R^{n}\right)\right)}:=C \epsilon_{\omega} \longrightarrow 0 \quad \text { as }|\omega| \longrightarrow \infty
$$

Using Hölder inequality, Sobolev embedding, and Lemma 7, we obtain

$$
\begin{aligned}
& \| \nabla\left(\left|u_{\omega}\right|^{8 /(n-4)} u_{\omega}\right. \\
& \left.-|u|^{8 /(n-4)} u\right) \|_{L^{2 n(n+4) /\left(n^{2}+4 n+8\right)}\left(0, t_{1} ; L^{2 n^{2}(n+4) /\left(n^{3}+8 n^{2}+16 n-32\right)}\left(R^{n}\right)\right)} \\
& \leq C\left\|\left|u_{\omega}\right|^{8 /(n-4)}\right\|_{L^{n(n+4) / 16}\left(0, t_{1} ; L^{2^{2}(n+4) / 4\left(n^{2}+4 n-16\right)}\left(R^{n}\right)\right)} \\
& \times\left\|\nabla\left(u_{\omega}-u\right)\right\|_{L^{2 n(n+4) /\left(n^{2}+4 n-24\right)}\left(0, t_{1} ; L^{2 n^{2}(n+4) /\left(n^{3}-16 n+96\right)}\left(R^{n}\right)\right)} \\
& +C\left(\left\|\left|u_{\omega}\right|^{(12-n) /(n-4)}\right\|_{L^{n(n+4) / 2(12-n)}\left(0, t_{1} ; L^{2 n(n+4) /(12-n)\left(n^{2}+4 n-16\right)}\left(R^{n}\right)\right)}\right. \\
& \left.+\left\||u|^{(12-n) /(n-4)}\right\|_{L^{n(n+4) / 2(12-n)}\left(0, t_{1} ; L^{2 n(n+4) /(12-n)\left(n^{2}+4 n-16\right)}\left(R^{n}\right)\right)}\right) \\
& \times\left\|u_{\omega}-u\right\|_{L^{n(n+4) / 2(n-4)}\left(0, t_{1} ; L^{2 n^{2}(n+4) /(n-4)\left(n^{2}+4 n-16\right)}\left(R^{n}\right)\right)} \\
& \times\|\nabla u\|_{L^{2 n(n+4) /\left(n^{2}+4 n-24\right)}\left(0, t_{1} ; L^{2 n^{2}(n+4) /\left(n^{3}-16 n+96\right)}\left(R^{n}\right)\right)} \\
& \leq C\left\|u_{\omega}\right\|_{X^{0}\left(0, t_{1}\right)}^{8 /(n-4)}\left\|\nabla\left(u_{\omega}-u\right)\right\|_{S^{0}\left(0, t_{1}\right)} \\
& +C\left(\left\|u_{\omega}\right\|_{X^{0}\left(0, t_{1}\right)}^{(12-n) /(n-4)}+\|u\|_{X^{0}\left(0, t_{1}\right)}^{(12-n) /(n-4)}\right) \\
& \times\left\|u_{\omega}-u\right\|_{X^{0}\left(0, t_{1}\right)}\|\nabla u\|_{S^{0}\left(0, t_{1}\right)} \\
& \leq C\left\|u_{\omega}\right\|_{X^{0}\left(0, t_{1}\right)}^{8 /(n-4)}\left\|\nabla\left(u_{\omega}-u\right)\right\|_{S^{0}\left(0, t_{1}\right)} \\
& +C\left\|u_{\omega}-u\right\|_{X^{0}\left(0, t_{1}\right)}^{8 /(n-4)}\|\nabla u\|_{S^{0}\left(0, t_{1}\right)} \\
& +C\|u\|_{X^{0}\left(0, t_{1}\right)}^{(12-n) /(n-4)}\left\|u_{\omega}-u\right\|_{X^{0}\left(0, t_{1}\right)}\|\nabla u\|_{S^{0}\left(0, t_{1}\right)} \\
& \leq C\left\|u_{\omega}-u\right\|_{X^{0}\left(0, t_{1}\right)}^{8 /(n-4)}\left\|\nabla\left(u_{\omega}-u\right)\right\|_{S^{0}\left(0, t_{1}\right)} \\
& +C\|u\|_{X^{0}\left(0, t_{1}\right)}^{8 /(n-4)}\left\|\nabla\left(u_{\omega}-u\right)\right\|_{S^{0}\left(0, t_{1}\right)} \\
& +C\left\|u_{\omega}-u\right\|_{X^{0}\left(0, t_{1}\right)}^{8 /(n-4)}\|\nabla u\|_{S^{0}\left(0, t_{1}\right)} \\
& +C\|u\|_{X^{0}\left(0, t_{1}\right)}^{(12-n) /(n-4)}\left\|u_{\omega}-u\right\|_{X^{0}\left(0, t_{1}\right)}\|\nabla u\|_{S^{0}\left(0, t_{1}\right)} \\
& \leq C\left\|u_{\omega}-u\right\|_{X^{0}\left(0, t_{1}\right)}^{8 /(n-4)}\left\|\nabla\left(u_{\omega}-u\right)\right\|_{S^{0}\left(0, t_{1}\right)} \\
& +C\left\|D^{2} u\right\|_{S^{0}\left(0, t_{1}\right)}^{8 /(n-4)}\left\|\nabla\left(u_{\omega}-u\right)\right\|_{S^{0}\left(0, t_{1}\right)} \\
& +C\left\|u_{\omega}-u\right\|_{X^{0}\left(0, t_{1}\right)}^{8 /(n-4)}\|\nabla u\|_{S^{0}\left(t_{0}, t_{1}\right)} \\
& +C\left\|D^{2} u\right\|_{S^{0}\left(0, t_{1}\right)}^{(12-n) /(n-4)}\left\|u_{\omega}-u\right\|_{X^{0}\left(0, t_{1}\right)}\|\nabla u\|_{S^{0}\left(0, t_{1}\right)} \\
& \leq C\left\|u_{\omega}-u\right\|_{X\left(0, t_{1}\right)}^{8 /(n-4)}\left\|\nabla\left(u_{\omega}-u\right)\right\|_{S^{0}\left(0, t_{1}\right)} \\
& +C\left\|D^{2} u\right\|_{S^{0}\left(0, t_{1}\right)}^{8 /(n-4)}\left\|\nabla\left(u_{\omega}-u\right)\right\|_{S^{0}\left(0, t_{1}\right)} \\
& +C\left\|u_{\omega}-u\right\|_{X\left(0, t_{1}\right)}^{8 /(n-4)}\|\nabla u\|_{S^{0}\left(0, t_{1}\right)} \\
& +C\left\|D^{2} u\right\|_{S^{0}\left(0, t_{1}\right)}^{(12-n) /(n-4)}\left\|u_{\omega}-u\right\|_{X\left(0, t_{1}\right)}\|\nabla u\|_{S^{0}\left(0, t_{1}\right)} .
\end{aligned}
$$


Substituting (53)-(54) into (51), we obtain

$$
\begin{aligned}
& \left\|\nabla\left(u_{\omega}-u\right)\right\|_{S^{0}\left(0, t_{1}\right)} \\
& \leq C\left\|\nabla\left(u_{\omega}(0)-u(0)\right)\right\|_{L^{2}\left(R^{n}\right)}+C \epsilon_{\omega} \\
& \quad+C\left\|u_{\omega}-u\right\|_{X\left(0, t_{1}\right)}^{8 /(n-4)}\left\|\nabla\left(u_{\omega}-u\right)\right\|_{S^{0}\left(0, t_{1}\right)} \\
& \quad+C\left\|D^{2} u\right\|_{S^{0}\left(0, t_{1}\right)}^{8 /(n-4)}\left\|\nabla\left(u_{\omega}-u\right)\right\|_{S^{0}\left(0, t_{1}\right)} \\
& \quad+C\left\|u_{\omega}-u\right\|_{X\left(0, t_{1}\right)}^{8 /(n-4)}\|\nabla u\|_{S^{0}\left(0, t_{1}\right)} \\
& \quad+C\left\|D^{2} u\right\|_{S^{0}\left(0, t_{1}\right)}^{(12-n) /(n-4)}\left\|u_{\omega}-u\right\|_{X\left(0, t_{1}\right)}\|\nabla u\|_{S^{0}\left(0, t_{1}\right)} \cdot
\end{aligned}
$$

Taking $t_{1}$ such that $C\left\|D^{2} u\right\|_{S^{0}\left(0, t_{1}\right)}^{8 /(n-4)} \leq 1 / 2, C\|\nabla u\|_{S^{0}\left(0, t_{1}\right)} \leq 1$, $C\left\|D^{2} u\right\|_{S^{0}\left(0, t_{1}\right)}^{(12-n) /(n-4)}\|\nabla u\|_{S^{0}\left(0, t_{1}\right)} \leq 1$, using Young's inequality and Lemma 7 , we obtain

$$
\begin{aligned}
& \left\|\nabla\left(u_{\omega}-u\right)\right\|_{S^{0}\left(0, t_{1}\right)} \\
& \leq 2 C\left\|\nabla\left(u_{\omega}(0)-u(0)\right)\right\|_{L^{2}\left(R^{n}\right)}+2 C \epsilon_{\omega} \\
& \quad+2 C\left\|u_{\omega}-u\right\|_{X\left(0, t_{1}\right)}^{8 /(n-4)}\left\|\nabla\left(u_{\omega}-u\right)\right\|_{S^{0}\left(0, t_{1}\right)} \\
& \quad+2\left\|u_{\omega}-u\right\|_{X\left(0, t_{1}\right)}^{8 /(n-4)}+2\left\|u_{\omega}-u\right\|_{X\left(0, t_{1}\right)} \\
& \leq 2 C\left\|\nabla\left(u_{\omega}(0)-u(0)\right)\right\|_{L^{2}\left(R^{n}\right)}+2 C \epsilon_{\omega} \\
& \quad+C\left\|u_{\omega}-u\right\|_{X\left(0, t_{1}\right)}^{(n+4) /(n-4)}+C\left\|\nabla\left(u_{\omega}-u\right)\right\|_{S^{0}\left(0, t_{1}\right)}^{8 /(n-4)} \\
& \quad+2\left\|u_{\omega}-u\right\|_{X\left(0, t_{1}\right)}^{8 /(n-4)}+2\left\|u_{\omega}-u\right\|_{X\left(0, t_{1}\right)} .
\end{aligned}
$$

Evidently, in order to get the estimate $\left\|\nabla\left(u_{\omega}-u\right)\right\|_{S^{0}\left(t_{0}, t_{1}\right)}$, we have to estimate the norm $\left\|u_{\omega}-u\right\|_{X\left(t_{0}, t_{1}\right)}$.

By (11)-(12), Lemmas 6 and 9, we obtain

$$
\begin{aligned}
& \left\|u_{\omega}-u\right\|_{X\left(0, t_{1}\right)} \\
& \leq C\left\|W(t)\left(u_{\omega}(0)-u(0)\right)\right\|_{X\left(0, t_{1}\right)} \\
& +C\left\|\left|u_{\omega}\right|^{8 /(n-4)} u_{\omega}-|u|^{8 /(n-4)} u\right\|_{Y\left(0, t_{1}\right)} \\
& \quad+\left\|\int_{0}^{t}(\theta(\omega s-I(\theta))) W(t)\left(|u|^{8 /(n-4)} u\right)(t) d t\right\|_{X\left(0, t_{1}\right)} \\
& \leq C\left\|D^{2}\left(u_{\omega}(0)-u(0)\right)\right\|_{L^{2}} \\
& \quad+C\left(\left\|u_{\omega}-u\right\|_{X\left(0, t_{1}\right)}^{4(n+8) /\left(n^{2}-16\right)}\left\|D^{2}\left(u_{\omega}-u\right)\right\|_{S^{0}\left(0, t_{1}\right)}^{4 n /\left(n^{2}-16\right)}\right. \\
& \left.\quad+\|u\|_{X\left(0, t_{1}\right)}^{4(n+8) /\left(n^{2}-16\right)}\left\|D^{2} u\right\|_{S^{0}\left(0, t_{1}\right)}^{4 n /\left(n^{2}-16\right)}\right)\left\|u_{\omega}-u\right\|_{X\left(0, t_{1}\right)} \\
& \quad+\left\|\int_{0}^{t}(\theta(\omega s)-I(\theta)) W(t) D^{2}\left(|u|^{8 /(n-4)} u\right) d t\right\|_{S^{0}\left(0, t_{1}\right)} .
\end{aligned}
$$

Let $\left\|\int_{0}^{t}(\theta(\omega s-I(\theta))) W(t) D^{2}\left(|u|^{8 /(n-4)} u\right) d t\right\|_{S^{0}\left(0, t_{1}\right)} \quad=$ $C \xi_{\omega}$. Noting that

$$
\begin{aligned}
& \left\|D^{2}\left(|u|^{8 /(n-4)} u\right)\right\|_{L^{\gamma^{\prime}}\left(0, t_{1} ; L^{\rho^{\prime}}\left(R^{n}\right)\right)} \\
& \leq C\left\||u|^{(12-n) /(n-4)}\right\|_{L^{2 n /(12-n)}\left(0, t_{1} ; L^{2 n^{2} /(n-4)(12-n)}\left(R^{n}\right)\right)} \\
& \times\|\nabla u\|_{L^{2 n /(n-4)}\left(0, t_{1} ; L^{2 n^{2} /\left(n^{2}-6 n+16\right)}\left(R^{n}\right)\right)}^{2} \\
& +C\left\||u|^{8 /(n-4)}\right\|_{L^{\gamma /(\gamma-2)}\left(0, t_{1} ; L^{\rho /(\rho-2)}\left(R^{n}\right)\right)}\left\|D^{2} u\right\|_{L^{\gamma}\left(0, t_{1} ; L^{\rho}\left(R^{n}\right)\right)} \\
& \leq C\|u\|_{L^{2 n /(n-4)}\left(0, t_{1} ; L^{\left.2 n^{2} /(n-4)^{2}\left(R^{n}\right)\right)}\right.}^{(12-n) /(n-4)}\left\|D^{2} u\right\|_{L^{\gamma}\left(0, t_{1} ; L^{\rho}\left(R^{n}\right)\right)}^{2} \\
& +C\left\||u|^{8 /(n-4)}\right\|_{L^{8 \gamma /(n-4)(\gamma-2)}\left(0, t_{1} ; L^{8 \rho /(n-4)(\rho-2)}\left(R^{n}\right)\right)} \\
& \times\left\|D^{2} u\right\|_{L^{\gamma}\left(0, t_{1} ; L^{\rho}\left(R^{n}\right)\right)} \leq C\left\|D^{2} u\right\|_{L^{\gamma}\left(0, t_{1} ; L^{\rho}\left(R^{n}\right)\right)}^{(n+4) /(n-4)},
\end{aligned}
$$

so $D^{2}\left(|u|^{8 /(n-4)} u\right) \in L^{\gamma^{\prime}}\left(0, t_{1} ; L^{\rho^{\prime}}\left(R^{n}\right)\right)$.

Using Lemma 10, we obtain

$$
C \xi_{\omega} \longrightarrow 0 \quad \text { as } \omega \longrightarrow \infty \text {. }
$$

From (57)-(59), we get

$$
\begin{aligned}
& \left\|u_{\omega}-u\right\|_{X\left(0, t_{1}\right)} \\
& \leq C\left\|D^{2}\left(u_{\omega}(0)-u(0)\right)\right\|_{L^{2}} \\
& +C\left(\left\|u_{\omega}-u\right\|_{X\left(0, t_{1}\right)}^{\left(n^{2}+4 n+16\right) /\left(n^{2}-16\right)}\left\|D^{2}\left(u_{\omega}-u\right)\right\|_{S^{0}\left(0, t_{1}\right)}^{4 n /\left(n^{2}-16\right)}\right. \\
& \left.+\left\|D^{2} u\right\|_{S^{0}\left(0, t_{1}\right)}^{8 /(n-4)}\left\|u_{\omega}-u\right\|_{X\left(0, t_{1}\right)}\right)+C \xi_{\omega} \\
& \leq C\left\|D^{2}\left(u_{\omega}(0)-u(0)\right)\right\|_{L^{2}} \\
& +C\left(\left\|D^{2} u_{\omega}\right\|_{S^{0}\left(0, t_{1}\right)}^{4 n /\left(n^{2}-16\right)}+\left\|D^{2} u\right\|_{S^{0}\left(0, t_{1}\right)}^{4 n /\left(n^{2}-16\right)}\right) \\
& \quad \times\left\|u_{\omega}-u\right\|_{X\left(0, t_{1}\right)}^{\left(n^{2}+4 n+16\right) /\left(n^{2}-16\right)}+C\left\|D^{2} u\right\|_{S^{0}\left(0, t_{1}\right)}^{8 /(n-4)} \\
& \quad \times\left\|u_{\omega}-u\right\|_{X\left(0, t_{1}\right)}+C \xi_{\omega} .
\end{aligned}
$$

Let $t_{1} \rightarrow 0$ such that $\left\|D^{2} u\right\|_{S^{0}\left(0, t_{1}\right)}^{8 /(n-4)} \leq 1 / 2$, thus we have

$$
\begin{aligned}
& \left\|u_{\omega}-u\right\|_{X\left(0, t_{1}\right)} \\
& \leq 2 C\left\|D^{2}\left(u_{\omega}(0)-u(0)\right)\right\|_{L^{2}} \\
& \quad+2 C\left(\left\|D^{2} u_{\omega}\right\|_{S^{0}\left(0, t_{1}\right)}^{4 n /\left(n^{2}-16\right)}+\left\|D^{2} u\right\|_{S^{0}\left(0, t_{1}\right)}^{4 n /\left(n^{2}-16\right)}\right) \\
& \quad \times\left\|u_{\omega}-u\right\|_{X\left(0, t_{1}\right)}^{\left(n^{2}+4 n+16\right) /\left(n^{2}-16\right)}+2 C \xi_{\omega} \\
& \leq 2 C\left\|D^{2}\left(u_{\omega}(0)-u(0)\right)\right\|_{L^{2}} \\
& \quad+2 C\left(2 M_{1}\right)^{4 n /\left(n^{2}-16\right)}\left\|u_{\omega}-u\right\|_{X\left(0, t_{1}\right)}^{\left(n^{2}+4 n+16\right) /\left(n^{2}-16\right)}+2 C \xi_{\omega},
\end{aligned}
$$

where $M_{1}=\max \left(\left\|D^{2} u\right\|_{S^{0}(0, l)},\left\|D^{2} u_{\omega}\right\|_{S^{0}(0, l)}\right)$. 
If $\left\|D^{2}\left(u_{\omega}(0)-u(0)\right)\right\|_{L^{2}}$ and $\xi_{\omega}$ are small such that

$$
\begin{aligned}
& (4 C)^{\left(n^{2}+4 n+16\right) /\left(n^{2}-16\right)}\left(2 M_{1}\right)^{4 n /\left(n^{2}-16\right)} \\
& \quad \times\left(\left\|D^{2}\left(u_{\omega}(0)-u(0)\right)\right\|_{L^{2}}+\xi_{\omega}\right)^{4(n+8) /\left(n^{2}-16\right)}<1,
\end{aligned}
$$

we obtain

$$
\left\|u_{\omega}-u\right\|_{X\left(0, t_{1}\right)} \leq 4 C\left\|D^{2}\left(u_{\omega}(0)-u(0)\right)\right\|_{L^{2}}+4 C \xi_{\omega} .
$$

Taking (63) into (56), we can get

$$
\begin{aligned}
\| \nabla & \left(u_{\omega}-u\right) \|_{S^{0}\left(0, t_{1}\right)} \\
\leq & 2 C\left\|\nabla\left(u_{\omega}(0)-u(0)\right)\right\|_{L^{2}\left(R^{n}\right)}+2 C \epsilon_{\omega} \\
& +\left(4 C\left\|D^{2}\left(u_{\omega}(0)-u(0)\right)\right\|_{L^{2}}+4 C \xi_{\omega}\right)^{(n+4) /(n-4)} \\
& +\left(4 C\left\|D^{2}\left(u_{\omega}(0)-u(0)\right)\right\|_{L^{2}}+4 C \xi_{\omega}\right)^{8 /(n-4)} \\
& +4 C\left\|D^{2}\left(u_{\omega}(0)-u(0)\right)\right\|_{L^{2}}+4 C \xi_{\omega} \\
& +C\left\|\nabla\left(u_{\omega}-u\right)\right\|_{S^{0}\left(0, t_{1}\right)}^{8 /(n-4)} .
\end{aligned}
$$

If $\left\|\nabla\left(u_{\omega}(0)-u(0)\right)\right\|_{L^{2}},\left\|D^{2}\left(u_{\omega}(0)-u(0)\right)\right\|_{L^{2}}$ and $\epsilon_{\omega}, \xi_{\omega}$ are small such that

$$
\begin{aligned}
C(2 C & \left\|\nabla\left(u_{\omega}(0)-u(0)\right)\right\|_{L^{2}\left(R^{n}\right)}+2 C \epsilon_{\omega} \\
& +\left(4 C\left\|D^{2}\left(u_{\omega}(0)-u(0)\right)\right\|_{L^{2}}+4 C \xi_{\omega}\right)^{(n+4) /(n-4)} \\
& +\left(4 C\left\|D^{2}\left(u_{\omega}(0)-u(0)\right)\right\|_{L^{2}}+4 C \xi_{\omega}\right)^{8 /(n-4)} \\
& \left.+4 C\left\|D^{2}\left(u_{\omega}(0)-u(0)\right)\right\|_{L^{2}}+4 C \xi_{\omega}\right)^{(12-n) /(n-4)}<\frac{1}{2},
\end{aligned}
$$

then we can obtain

$$
\begin{aligned}
& \left\|\nabla\left(u_{\omega}-u\right)\right\|_{S^{0}\left(0, t_{1}\right)} \\
& \leq 4 C\left\|\nabla\left(u_{\omega}(0)-u(0)\right)\right\|_{L^{2}\left(R^{n}\right)}+4 C \epsilon_{\omega} \\
& \quad+2\left(4 C\left\|D^{2}\left(u_{\omega}(0)-u(0)\right)\right\|_{L^{2}}+4 C \xi_{\omega}\right)^{(n+4) /(n-4)} \\
& \quad+2\left(4 C\left\|D^{2}\left(u_{\omega}(0)-u(0)\right)\right\|_{L^{2}}+4 C \xi_{\omega}\right)^{8 /(n-4)} \\
& \quad+8 C\left\|D^{2}\left(u_{\omega}(0)-u(0)\right)\right\|_{L^{2}}+8 C \xi_{\omega} .
\end{aligned}
$$

So we have

$\left\|\nabla\left(u_{\omega}(x, t)-u(x, t)\right)\right\|_{L^{q}\left(0, t_{1} ; L^{r}\left(R^{n}\right)\right)} \longrightarrow 0 \quad$ as $|\omega| \longrightarrow \infty$.

Let $T_{1}=\sup \left\{t \mid 0<t<l,\left\|\nabla\left(u_{\omega}-u\right)\right\|_{L^{q}\left(0, t ; L^{r}\left(R^{n}\right)\right)} \rightarrow\right.$ 0 as $|\omega| \rightarrow \infty\}$. By continuous extension method and contradiction method, we can prove that $T_{1}=l$.

(3) At last, we discuss the estimate $\left\|D^{2}\left(u_{\omega}-u\right)\right\|_{L^{q}\left(0, l ; L^{r}\left(R^{n}\right)\right)}$.
As in Lemmas 8 and 9, we define $F(u)=|u|^{8 /(n-4)} u$. By (11) and (12), we have

$$
D^{2}\left(u_{\omega}-u\right)=i\left(K_{1}+K_{2}+K_{3}\right)
$$

where $K_{1}=\int_{0}^{t} \theta(\omega s) W(t-s) A_{1}\left(u_{\omega}, u\right) d s, K_{2}=\int_{0}^{t} \theta(\omega s) W(t-$ s) $A_{2}\left(u_{\omega}, u\right) d s, K_{3}=\int_{0}^{t}[\theta(\omega s)-I(\theta)] W(t-s) A_{3}(u) d s$, and $A_{1}\left(u_{\omega}, u\right), A_{2}\left(u_{\omega}, u\right)$, and $A_{3}(u)$ are as follows:

$$
\begin{aligned}
A_{1}\left(u_{\omega}, u\right)= & F^{\prime}\left(u_{\omega}\right) D^{2}\left(u_{\omega}-u\right)+\left(D u_{\omega}-D u\right)^{\perp} \\
& \times F^{\prime \prime}\left(u_{\omega}\right) D\left(u_{\omega}\right), \\
A_{2}\left(u_{\omega}, u\right)= & (D u)^{\perp} \times\left[F^{\prime \prime}\left(u_{\omega}\right) D u_{\omega}-F^{\prime \prime}(u) D u\right] \\
& +\left[F^{\prime}\left(u_{\omega}\right)-F^{\prime}(u)\right] D^{2} u, \\
A_{3}(u)= & (D u)^{\perp} \times F^{\prime \prime}(u) D u+F^{\prime}(u) D^{2} u
\end{aligned}
$$

are all $n \times n$ matrixes.

Using (11)-(12) and Lemma 5, we obtain

$$
\begin{aligned}
& \left\|D^{2}\left(u_{\omega}-u\right)\right\|_{S^{0}\left(0, t_{1}\right)} \\
& \leq C\left\|D^{2}\left(u_{\omega}(0)-u(0)\right)\right\|_{L^{2}\left(R^{n}\right)} \\
& +C\left(\left\|K_{1}\right\|_{L^{q}\left(0, t_{1} ; L^{r}\left(R^{n}\right)\right)}+\left\|K_{2}\right\|_{L^{q}\left(0, t_{1} ; L^{r}\left(R^{n}\right)\right)}\right. \\
& \left.+\left\|K_{3}\right\|_{L^{q}\left(0, t_{1} ; L^{r}\left(R^{n}\right)\right)}\right) \\
& \leq C\left\|D^{2}\left(u_{\omega}(0)-u(0)\right)\right\|_{L^{2}\left(R^{n}\right)} \\
& +C\left(\left\|A_{1}\right\|_{L^{\gamma^{\prime}}\left(0, t_{1} ; L^{\rho^{\prime}}\left(R^{n}\right)\right)}+\left\|A_{2}\right\|_{L^{\gamma^{\prime}}\left(0, t_{1} ; L^{\rho^{\prime}}\left(R^{n}\right)\right)}\right. \\
& \left.+\left\|A_{3}\right\|_{L^{\gamma^{\prime}}\left(0, t_{1} ; L^{\rho^{\prime}}\left(R^{n}\right)\right)}\right) .
\end{aligned}
$$

For the case $5 \leq n<12$, using Hölder inequality and Sobolev embedding, we have

$$
\begin{aligned}
& \left\|(D u)^{\perp} \times F^{\prime \prime}(u) D u\right\|_{L^{\gamma^{\prime}}\left(0, t_{1} ; L^{\rho^{\prime}}\left(R^{n}\right)\right)} \\
& \leq C\left\||u|^{(12-n) /(n-4)}\right\|_{L^{2 n /(12-n)}\left(0, t_{1} ; L^{2 n^{2} /(n-4)(12-n)}\left(R^{n}\right)\right)} \\
& \quad \times\|D u\|_{L^{2 n /(n-4)}\left(0, t_{1} ; L^{2 n^{2} /\left(n^{2}-6 n+16\right)}\left(R^{n}\right)\right)}^{2} \\
& \leq\|u\|_{L^{2 n /(n-4)}\left(0, t_{1} ; L^{\left.2 n^{2} /(n-4)^{2}\left(R^{n}\right)\right)}\right.}^{(12-n) /(n-4)}\left\|D^{2} u\right\|_{L^{\gamma}\left(0, t_{1} ; L^{\rho}\left(R^{n}\right)\right)}^{2} \\
& \leq C\left\|D^{2} u\right\|_{L^{\gamma}\left(0, t_{1} ; L^{\rho}\left(R^{n}\right)\right)}^{(n+4) /(n-4)},
\end{aligned}
$$




$$
\begin{aligned}
& \left\|F^{\prime}(u) D^{2} u\right\|_{L^{\gamma^{\prime}\left(0, t_{1} ; L^{\rho^{\prime}}\left(R^{n}\right)\right)}}
\end{aligned}
$$

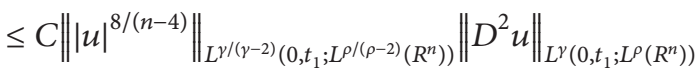

$$
\begin{aligned}
& =C\|u\|_{L^{8 /\left(q^{2}-2\right)(n-4)}\left(0, t_{1} ; L^{8 \rho /(\rho-2)(n-4)}\left(R^{n}\right)\right)}^{8 /(n-4)}\left\|D^{2} u\right\|_{L^{\gamma}\left(0, t_{1} ; L^{\rho}\left(R^{n}\right)\right)} \\
& \leq\left\|D^{2} u\right\|_{L^{\gamma}\left(0, t_{1} ; L^{\rho}\left(R^{n}\right)\right)}^{(n+4) /(n-4)} \text {. }
\end{aligned}
$$

Since $u \in L^{\gamma}\left(0, l ; H^{2, \rho}\left(R^{n}\right)\right)$, we have $A_{3}(u) \in L^{\gamma^{\prime}}\left(0, t_{1}\right.$; $\left.L^{\rho^{\prime}}\left(R^{n}\right)\right)$; thus by Lemma 10 ,

$\left\|A_{3}(u)\right\|_{L^{y^{\prime}}\left(0, t_{1} ; L^{\rho^{\prime}}\left(R^{n}\right)\right)}:=C \zeta_{\omega} \longrightarrow 0 \quad$ as $\omega \longrightarrow \infty$.

In the following, we analyze the norm $\| A_{1}\left(u_{\omega}\right.$,

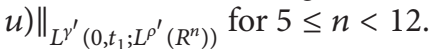
obtain

Using Hölder inequality and Sobolev embedding, we

$$
\begin{aligned}
& \left\|\left(D u_{\omega}-D u\right)^{\perp} \times F^{\prime \prime}\left(u_{\omega}\right) D u_{\omega}\right\|_{L^{\nu^{\prime}}\left(0, t_{1} ; L^{\rho^{\prime}}\left(R^{n}\right)\right)} \\
& \leq C\left\|D u_{\omega}-D u\right\|_{L^{v}\left(0, t_{1}: L^{\rho_{1}}\left(R^{n}\right)\right)} \\
& \times\left\|\left|u_{\omega}\right|^{(12-n) /(n-4)}\right\|_{L^{a}\left(0, t_{1} ; L^{b}\left(R^{n}\right)\right)}\left\|D u_{\omega}\right\|_{L^{\gamma}\left(0, t_{1} ; L^{\rho_{1}}\left(R^{n}\right)\right)} \\
& \leq C\left\|D u_{\omega}-D u\right\|_{L^{\gamma}\left(0, t_{1} ; L^{\rho_{1}}\left(R^{n}\right)\right)}\left\|u_{\omega}\right\|_{L^{\gamma}\left(0, t_{1} ; L^{(12-n) b /(n-4)}\left(R^{n}\right)\right)}^{(12-n) /(n-4)} \\
& \times\left\|D u_{\omega}\right\|_{L^{\gamma}\left(0, t_{1} ; L^{\rho_{1}}\left(R^{n}\right)\right)} \\
& \leq C\left\|D^{2}\left(u_{\omega}-u\right)\right\|_{L^{\gamma}\left(0, t_{1} ; L^{P}\left(R^{n}\right)\right)}\left\|D^{2} u_{\omega}\right\|_{L^{\gamma}\left(0, t_{1} ; L^{P}\left(R^{n}\right)\right)}^{(12-n) /(n-4)} \\
& \times\left\|D^{2} u_{\omega}\right\|_{L^{\gamma}\left(0, t_{1} ; L^{\rho}\left(R^{n}\right)\right)} \\
& \leq C\left\|D^{2}\left(u_{\omega}-u\right)\right\|_{L^{\gamma}\left(0, t_{1} ; L^{P}\left(R^{n}\right)\right)}^{(n+4) /(n-4)} \\
& +C\left\|D^{2} u\right\|_{L^{\gamma}\left(0, t_{1} ; L^{\rho}\left(R^{n}\right)\right)}^{8 /(n-4)}\left\|D^{2}\left(u_{\omega}-u\right)\right\|_{L^{\gamma}\left(0, t_{1} ; L^{\rho}\left(R^{n}\right)\right)},
\end{aligned}
$$

where $a=\gamma /(\gamma-3), b=n(n-4) \rho /(n-2 \rho)(12-n), \rho_{1}=$ $n \rho /(n-\rho)$.

Similarly, we can get

$$
\begin{aligned}
& \left\|F^{\prime}\left(u_{\omega}\right) D^{2}\left(u_{\omega}-u\right)\right\|_{L^{\gamma^{\prime}}\left(0, t_{1} ; L^{\rho^{\prime}}\left(R^{n}\right)\right)} \\
& \leq C\left\|D^{2}\left(u_{\omega}-u\right)\right\|_{L^{\gamma}\left(0, t_{1} ; L^{\rho}\left(R^{n}\right)\right)}^{(n+4) /(n-4)} \\
& \quad+C\left\|D^{2} u\right\|_{L^{\gamma}\left(0, t_{1} ; L^{\rho}\left(R^{n}\right)\right)}^{8 /(n-)}\left\|D^{2}\left(u_{\omega}-u\right)\right\|_{L^{\gamma}\left(0, t_{1} ; L^{\rho}\left(R^{n}\right)\right)} .
\end{aligned}
$$

Combing (74) and (75), we have

$$
\begin{aligned}
& \left\|A_{1}\left(u_{\omega}, u\right)\right\|_{L^{\gamma^{\prime}}\left(0, t_{1} ; L^{\rho^{\prime}}\left(R^{n}\right)\right)} \\
& \leq C\left\|D^{2}\left(u_{\omega}-u\right)\right\|_{L^{\gamma}\left(0, t_{1} ; L^{\rho}\left(R^{n}\right)\right)}^{(n+4) /(n-4)} \\
& \quad+C\left\|D^{2} u\right\|_{L^{\gamma}\left(0, t_{1} ; L^{\rho}\left(R^{n}\right)\right)}^{8 /(n-4)}\left\|D^{2}\left(u_{\omega}-u\right)\right\|_{L^{\gamma}\left(0, t_{1} ; L^{\rho}\left(R^{n}\right)\right)} .
\end{aligned}
$$

At last, we analyze the norm $\left\|A_{2}\left(u_{\omega}, u\right)\right\|_{L^{y^{\prime}}\left(0, t_{1} ; L^{\rho^{\prime}}\left(R^{n}\right)\right)}$. We divide it into two cases.

Case I (5 $\leq n \leq 8)$. Using Hölder inequality, Sobolev embedding, and (73), we can get

$$
\begin{aligned}
& \left\|(D u)^{\perp} \times\left[F^{\prime \prime}\left(u_{\omega}\right) D u_{\omega}-F^{\prime \prime}(u) D u\right]\right\|_{L^{\gamma^{\prime}}\left(0, t_{1} ; L^{\rho^{\prime}}\left(R^{n}\right)\right)} \\
& \leq\left\|(D u)^{\perp} \times F^{\prime \prime}\left(u_{\omega}\right) D\left(u_{\omega}-u\right)\right\|_{L^{\prime}\left(0, t_{1} ; L^{\prime}\left(R^{n}\right)\right)}
\end{aligned}
$$

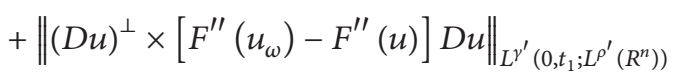

$$
\begin{aligned}
& \leq C\left\|D^{2} u\right\|_{L^{\gamma}\left(0, t_{1} ; L^{\rho}\left(R^{n}\right)\right)}\left\|D^{2} u_{\omega}\right\|_{L^{\gamma}\left(0, t_{1} ; L^{\rho}\left(R^{n}\right)\right)}^{(12-n) /(n-4)} \\
& \times\left\|D^{2}\left(u_{\omega}-u\right)\right\|_{L^{\gamma}\left(0, t_{1} ; L^{\rho}\left(R^{n}\right)\right)} \\
& +C \|(D u)^{\perp} \times\left(\left|u_{\omega}\right|^{2(8-n) /(n-4)}+|u|^{2(8-n) /(n-4)}\right) \\
& \times\left|u_{\omega}-u\right| D u \|_{L^{\prime}\left(0, t_{1} ; L^{\rho^{\prime}}\left(R^{n}\right)\right)} \\
& \leq C\left\|D^{2} u\right\|_{L^{\gamma}\left(0, t_{1} ; L^{\rho}\left(R^{n}\right)\right)}\left\|D^{2} u_{\omega}\right\|_{L^{\gamma}\left(0, t_{1} ; L^{\rho}\left(R^{n}\right)\right)}^{(12-n) /(n-4)} \\
& \times\left\|D^{2}\left(u_{\omega}-u\right)\right\|_{L^{\gamma}\left(0, t_{1} ; L^{\rho}\left(R^{n}\right)\right)} \\
& +C\left(\left\|\left|u_{\omega}\right|^{2(8-n) /(n-4)}\right\|_{L^{8 /(8-n)}\left(0, t_{1} ; L^{n^{2}((n-4))(8-n)}\left(R^{n}\right)\right)}\right. \\
& \left.+\left\||u|^{2(8-n) /(n-4)}\right\|_{L^{8 /(8-n)}\left(0, t_{1} ; L^{n^{2} /(n-4)(8-n)}\left(R^{n}\right)\right)}\right) \\
& \times\left\|u_{\omega}-u\right\|_{L^{2 n /(n-4)}\left(0, t_{1} ; L^{2 n^{2} /\left(n^{2}-8 n+16\right)}\left(R^{n}\right)\right)} \\
& \times\|D u\|_{L^{2 n /(n-4)}\left(0, t_{1} ; L^{2 n^{2} /\left(n^{2}-6 n+16\right)}\left(R^{n}\right)\right)}^{2} \\
& \leq C\left\|D^{2} u\right\|_{L^{\gamma}\left(0, t_{1} ; L^{\rho}\left(R^{n}\right)\right)}\left\|D^{2} u_{\omega}\right\|_{L^{\gamma}\left(0, t_{1} ; L^{\rho}\left(R^{n}\right)\right)}^{(12-n) /(n-4)} \\
& \times\left\|D^{2}\left(u_{\omega}-u\right)\right\|_{L^{\gamma}\left(0, t_{1} ; L^{P}\left(R^{n}\right)\right)} \\
& +C\left(\left\|D^{2} u_{\omega}\right\|_{L^{\gamma}\left(0, t_{1} ; L^{\rho}\left(R^{n}\right)\right)}^{2(8-n) /(n-4)}+\left\|D^{2} u\right\|_{L^{\gamma}\left(0, t_{1} ; L^{\rho}\left(R^{n}\right)\right)}^{2(8-n) /(n-4)}\right) \\
& \times\left\|D^{2}\left(u_{\omega}-u\right)\right\|_{L^{\gamma}\left(0, t_{1} ; L^{\rho}\left(R^{n}\right)\right)}\left\|D^{2} u\right\|_{L^{\gamma}\left(0, t_{1} ; L^{\rho}\left(R^{n}\right)\right)}^{2} \\
& \leq C\left\|D^{2}\left(u_{\omega}-u\right)\right\|_{L^{\gamma}\left(0, t_{1} ; L^{\rho}\left(R^{n}\right)\right)}^{8 /(n-4)}\left\|D^{2} u\right\|_{L^{\gamma}\left(0, t_{1} ; L^{\rho}\left(R^{n}\right)\right)} \\
& +C\left\|D^{2}\left(u_{\omega}-u\right)\right\|_{L^{\gamma}\left(0, t_{1} ; L^{\rho}\left(R^{n}\right)\right)}\left\|D^{2} u\right\|_{L^{\gamma}\left(0, t_{1} ; L^{\rho}\left(R^{n}\right)\right)}^{8 /(n-4)} \\
& +C\left\|D^{2}\left(u_{\omega}-u\right)\right\|_{L^{\gamma}\left(0, t_{1} ; L^{(}\left(R^{n}\right)\right)}^{(12-n) /(n-4)}\left\|D^{2} u\right\|_{L^{\gamma}\left(0, t_{1} ; L^{\rho}\left(R^{n}\right)\right)}^{2} .
\end{aligned}
$$

Similarly, we can get

$$
\begin{aligned}
& \left\|\left[F^{\prime}\left(u_{\omega}\right)-F^{\prime}(u)\right] D^{2} u\right\|_{L^{y^{\prime}}\left(0, t_{1} ; L^{\rho^{\prime}}\left(R^{n}\right)\right)} \\
& \leq C \|\left|u_{\omega}-u\right|\left(\left|u_{\omega}\right|^{(12-n) /(n-4)}+|u|^{(12-n) /(n-4)}\right) \\
& \times D^{2} u \|_{L^{y^{\prime}}\left(0, t_{1} ; L^{\rho^{\prime}}\left(R^{n}\right)\right)}
\end{aligned}
$$


10

Abstract and Applied Analysis

$$
\begin{aligned}
& \leq C\left(\left\|\left|u_{\omega}\right|^{(12-n) /(n-4)}\right\|_{L^{12 n /(12-n)}\left(0, t_{1} ; L^{2 n^{2} /\left(n^{2}-8 n+16\right)}\left(R^{n}\right)\right)}\right. \\
& \left.\quad+\left\||u|^{(12-n) /(n-4)}\right\|_{L^{12 n /(12-n)}\left(0, t_{1} ; L^{2 n^{2} /\left(n^{2}-8 n+16\right)}\left(R^{n}\right)\right)}\right) \\
& \times\left\|u_{\omega}-u\right\|_{L^{2 n /(n-4)}\left(0, t_{1} ; L^{2 n^{2} /\left(n^{2}-8 n+16\right)}\left(R^{n}\right)\right)}\left\|D^{2} u\right\|_{L^{\gamma}\left(0, t_{1} ; L^{\rho}\left(R^{n}\right)\right)} \\
& \leq C\left(\left\|D^{2} u_{\omega}\right\|_{L^{\gamma}\left(0, t_{1} ; L^{\rho}\left(R^{n}\right)\right)}^{(12-n) /(n-4)}+\left\|D^{2} u\right\|_{L^{\gamma}\left(0, t_{1} ; L^{\rho}\left(R^{n}\right)\right)}^{(12-n) /(n-4)}\right) \\
& \quad \times\left\|D^{2}\left(u_{\omega}-u\right)\right\|_{L^{\gamma}\left(0, t_{1} ; L^{\rho}\left(R^{n}\right)\right)}\left\|D^{2} u\right\|_{L^{\gamma}\left(0, t_{1} ; L^{\rho}\left(R^{n}\right)\right)} \\
& \leq C\left\|D^{2}\left(u_{\omega}-u\right)\right\|_{L^{\gamma}\left(0, t_{1} ; L^{\rho}\left(R^{n}\right)\right)}^{8 /(n-4)}\left\|D^{2} u\right\|_{L^{\gamma}\left(0, t_{1} ; L^{\rho}\left(R^{n}\right)\right)}\|\|^{2} u \|_{L^{\gamma}\left(0, t_{1} ; L^{\rho}\left(R^{n}\right)\right)}^{8 /(n-4)}
\end{aligned}
$$

Combing (77) and (78), we can obtain

$$
\begin{aligned}
& \left\|A_{2}\left(u_{\omega}, u\right)\right\|_{L^{\gamma^{\prime}}\left(0, t_{1} ; L^{\rho^{\prime}}\left(R^{n}\right)\right)} \\
& \leq C\left\|D^{2}\left(u_{\omega}-u\right)\right\|_{L^{\gamma}\left(0, t_{1} ; L^{\rho}\left(R^{n}\right)\right)}^{8 /(n-4)}\left\|D^{2} u\right\|_{L^{\gamma}\left(0, t_{1} ; L^{\rho}\left(R^{n}\right)\right)} \\
& \quad+C\left\|D^{2}\left(u_{\omega}-u\right)\right\|_{L^{\gamma}\left(0, t_{1} ; L^{\rho}\left(R^{n}\right)\right)}\left\|D^{2} u\right\|_{L^{\gamma}\left(0, t_{1} ; L^{\rho}\left(R^{n}\right)\right)}^{8 /(n-4)} \\
& \quad+C\left\|D^{2}\left(u_{\omega}-u\right)\right\|_{L^{\gamma}\left(0, t_{1} ; L^{\rho}\left(R^{n}\right)\right)}^{(12-n) /(n-4)}\left\|D^{2} u\right\|_{L^{\gamma}\left(0, t_{1} ; L^{\rho}\left(R^{n}\right)\right)}^{2} .
\end{aligned}
$$

From (70), (73), (76), and (79), we have

$$
\begin{aligned}
& \left\|D^{2}\left(u_{\omega}-u\right)\right\|_{S^{0}\left(0, t_{1}\right)} \\
& \leq C\left\|D^{2}\left(u_{\omega}(0)-u(0)\right)\right\|_{L^{2}\left(R^{n}\right)}+C \zeta_{\omega} \\
& \quad+C\left\|D^{2}\left(u_{\omega}-u\right)\right\|_{L^{\gamma}\left(0, t_{1} ; L^{\rho}\left(R^{n}\right)\right)}^{8 /(n-4)}\left\|D^{2} u\right\|_{L^{\gamma}\left(0, t_{1} ; L^{\rho}\left(R^{n}\right)\right)} \\
& \quad+C\left\|D^{2}\left(u_{\omega}-u\right)\right\|_{L^{\gamma}\left(0, t_{1} ; L^{\rho}\left(R^{n}\right)\right)}\left\|D^{2} u\right\|_{L^{\gamma}\left(0, t_{1} ; L^{\rho}\left(R^{n}\right)\right)}^{8 /(n-4)} \\
& \quad+C\left\|D^{2}\left(u_{\omega}-u\right)\right\|_{L^{\gamma}\left(0, t_{1} ; L^{\rho}\left(R^{n}\right)\right)}^{(n+4) /(n-4)} \\
& \quad+C\left\|D^{2}\left(u_{\omega}-u\right)\right\|_{L^{\gamma}\left(0, t_{1} ; L^{\rho}\left(R^{n}\right)\right)}^{(12-n) /(n-4)}\left\|D^{2} u\right\|_{L^{\gamma}\left(0, t_{1} ; L^{\rho}\left(R^{n}\right)\right)}^{2} .
\end{aligned}
$$

Taking $C\left\|D^{2} u\right\|_{L^{\gamma}\left(0, t_{1} ; L^{\rho}\left(R^{n}\right)\right)}^{8 /(n-4)}<1 / 2$, we can get

$$
\begin{aligned}
& \left\|D^{2}\left(u_{\omega}-u\right)\right\|_{S^{0}\left(0, t_{1}\right)} \\
& \leq 2 C\left\|D^{2}\left(u_{\omega}(0)-u(0)\right)\right\|_{L^{2}\left(R^{n}\right)}+2 C \zeta_{\omega} \\
& \quad+2 C\left\|D^{2}\left(u_{\omega}-u\right)\right\|_{L^{\gamma}\left(0, t_{1} ; L^{\rho}\left(R^{n}\right)\right)}^{8 /(n-4)}\left\|D^{2} u\right\|_{L^{\gamma}\left(0, t_{1} ; L^{\rho}\left(R^{n}\right)\right)} \\
& \quad+2 C\left\|D^{2}\left(u_{\omega}-u\right)\right\|_{L^{\gamma}\left(0, t_{1} ; L^{\rho}\left(R^{n}\right)\right)}^{(n+4) /(n-4)} \\
& \quad+2 C\left\|D^{2}\left(u_{\omega}-u\right)\right\|_{L^{\gamma}\left(0, t_{1} ; L^{\rho}\left(R^{n}\right)\right)}^{(12-n) /(n-4)}\left\|D^{2} u\right\|_{L^{\gamma}\left(0, t_{1} ; L^{\rho}\left(R^{n}\right)\right)}^{2} .
\end{aligned}
$$

Furthermore, if $\left\|D^{2}\left(u_{\omega}(0)-u(0)\right)\right\|_{L^{2}\left(R^{n}\right)}$ and $\zeta_{\omega}$ are small enough such that

$$
\begin{aligned}
(2 C)^{(12-n) / 8}(4 C)^{(12-n) /(n-4)} & \\
& \times\left(\left\|D^{2}\left(u_{\omega}(0)-u(0)\right)\right\|_{L^{2}\left(R^{n}\right)}+\zeta_{\omega}\right)^{(12-n) /(n-4)} \\
& +(2 C)^{(8-n) / 4}(4 C)^{2(8-n) /(n-4)} \\
& \times\left(\left\|D^{2}\left(u_{\omega}(0)-u(0)\right)\right\|_{L^{2}\left(R^{n}\right)}+\zeta_{\omega}\right)^{2(8-n) /(n-4)} \\
& +2 C(4 C)^{8 /(n-4)}\left(\left\|D^{2}\left(u_{\omega}(0)-u(0)\right)\right\|_{L^{2}\left(R^{n}\right)}+\zeta_{\omega}\right)^{8 /(n-4)} \\
< & \frac{1}{2}
\end{aligned}
$$

then we have

$$
\left\|D^{2}\left(u_{\omega}-u\right)\right\|_{S^{0}\left(0, t_{1}\right)} \leq 4 C\left\|D^{2}\left(u_{\omega}(0)-u(0)\right)\right\|_{L^{2}\left(R^{n}\right)}+4 C \zeta_{\omega} .
$$

Case II $(8<n<12)$. Noting that

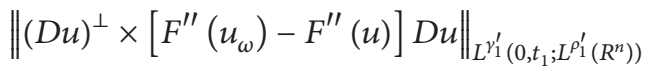

$$
\begin{aligned}
& \leq C\left\|\left|u_{\omega}-u\right|^{(12-n) /(n-4)}\right\|_{L^{n(n+4) / 2(12-n)}\left(0, t_{1} ; L^{2 n^{2}(n+4) /(12-n)\left(n^{2}+4 n-16\right)}\left(R^{n}\right)\right)} \\
& \times\|D u\|_{L^{q}\left(0, t_{1} ; L^{p}\left(R^{n}\right)\right)}^{2} \\
& \leq C\left\|u_{\omega}-u\right\|_{X_{0}\left(0, t_{1}\right)}^{(12-n) /(n-4)}\left\|D^{2} u\right\|_{L^{q}\left(0, t_{1} ; L^{p_{1}}\left(R^{n}\right)\right)}^{2} \\
& \leq C\left\|u_{\omega}-u\right\|_{X\left(0, t_{1}\right)}^{(12-n) /(n-4)}\left\|D^{2} u\right\|_{S^{0}\left(0, t_{1}\right)}^{2},
\end{aligned}
$$

where $\gamma_{1}^{\prime}=2 n(n+4) /\left(n^{2}+4 n+8\right), \rho_{1}^{\prime}=2 n^{2}(n+4) /\left(n^{3}+8 n^{2}+\right.$ $16 n-32), q=4 n(n+4) /\left(n^{2}+8 n-40\right), p=2 n^{2}(n+4) /\left(n^{3}-\right.$ $24 n+80)$, and $p_{1}=2 n^{2}(n+4) /\left(n^{3}+2 n^{2}-16 n+80\right)$; thus we have

$$
\begin{aligned}
& \left\|(D u)^{\perp} \times\left[F^{\prime \prime}\left(u_{\omega}\right) D u_{\omega}-F^{\prime \prime}(u) D u\right]\right\|_{L^{\gamma_{1}^{\prime}}\left(0, t_{1} ; L^{\rho_{1}^{\prime}}\left(R^{n}\right)\right)} \\
& \leq\left\|(D u)^{\perp} \times F^{\prime \prime}\left(u_{\omega}\right) D\left(u_{\omega}-u\right)\right\|_{L_{1}^{\gamma_{1}^{\prime}}\left(0, t_{1} ; L^{\prime}{ }_{1}^{\prime}\left(R^{n}\right)\right)} \\
& +\left\|(D u)^{\perp} \times\left[F^{\prime \prime}\left(u_{\omega}\right)-F^{\prime \prime}(u)\right] D u\right\|_{L^{\gamma_{1}^{\prime}}\left(0, t_{1} ; L^{\rho_{1}^{\prime}}\left(R^{n}\right)\right)} \\
& \leq C\left\|D^{2} u\right\|_{S^{0}\left(0, t_{1}\right)}\left\|u_{\omega}\right\|_{X\left(0, t_{1}\right)}^{(12-n) /(n-4)}\left\|D^{2}\left(u_{\omega}-u\right)\right\|_{S^{0}\left(0, t_{1}\right)} \\
& +C\left\|D^{2} u\right\|_{S^{0}\left(0, t_{1}\right)}^{2}\left\|u_{\omega}-u\right\|_{X\left(0, t_{1}\right)}^{(12-n) /(n-4)} \\
& \leq C\left\|D^{2} u\right\|_{S^{0}\left(0, t_{1}\right)}\left\|D^{2}\left(u_{\omega}-u\right)\right\|_{S^{0}\left(0, t_{1}\right)}^{8 /(n-4)} \\
& +C\left\|D^{2} u\right\|_{S^{0}\left(0, t_{1}\right)}^{8 /(n-4)}\left\|D^{2}\left(u_{\omega}-u\right)\right\|_{S^{0}\left(0, t_{1}\right)} \\
& +C\left\|D^{2} u\right\|_{S^{0}\left(0, t_{1}\right)}^{2}\left\|u_{\omega}-u\right\|_{X\left(0, t_{1}\right)}^{(12-n) /(n-4)} .
\end{aligned}
$$


Combing (78) and (85), we can get

$$
\begin{aligned}
& \left\|A_{2}\left(u_{\omega}, u\right)\right\|_{L^{\gamma_{1}^{\prime}\left(0, t_{1} ; L^{\rho_{1}^{\prime}}\left(R^{n}\right)\right)}} \\
& \leq C\left\|D^{2} u\right\|_{S^{0}\left(0, t_{1}\right)}\left\|D^{2}\left(u_{\omega}-u\right)\right\|_{S^{0}\left(0, t_{1}\right)}^{8 /(n-4)} \\
& +C\left\|D^{2} u\right\|_{S^{0}\left(0, t_{1}\right)}^{8 /(n-4)}\left\|D^{2}\left(u_{\omega}-u\right)\right\|_{S^{0}\left(0, t_{1}\right)} \\
& +C\left\|D^{2} u\right\|_{S^{0}\left(0, t_{1}\right)}^{2}\left\|u_{\omega}-u\right\|_{X\left(0, t_{1}\right)}^{(12-n) /(n-4)} .
\end{aligned}
$$

From (70), (73), (76), and (86), we have

$$
\begin{aligned}
& \left\|D^{2}\left(u_{\omega}-u\right)\right\|_{S^{0}\left(0, t_{1}\right)} \\
& \leq C\left\|D^{2}\left(u_{\omega}(0)-u(0)\right)\right\|_{L^{2}\left(R^{n}\right)} \\
& \quad+C\left\|D^{2}\left(u_{\omega}-u\right)\right\|_{S^{0}\left(0, t_{1}\right)}^{(n+4) /(n-4)} \\
& \quad+C\left\|D^{2} u\right\|_{S^{0}\left(0, t_{1}\right)}\left\|D^{2}\left(u_{\omega}-u\right)\right\|_{S^{0}\left(0, t_{1}\right)}^{8 /(n-4)} \\
& \quad+C\left\|D^{2} u\right\|_{S^{0}\left(0, t_{1}\right)}^{8 /(n-4)}\left\|D^{2}\left(u_{\omega}-u\right)\right\|_{S^{0}\left(0, t_{1}\right)} \\
& \quad+C\left\|D^{2} u\right\|_{S^{0}\left(0, t_{1}\right)}^{2}\left\|u_{\omega}-u\right\|_{X\left(0, t_{1}\right)}^{(12-n) /(n-4)}+C \zeta_{\omega} .
\end{aligned}
$$

Taking $C\left\|D^{2} u\right\|_{L^{\gamma}\left(0, t_{1} ; L^{\rho}\left(R^{n}\right)\right)}^{8 /(n-4)}<1 / 2, C\left\|D^{2} u\right\|_{S^{0}\left(0, t_{1}\right)} \leq 1$, and $C\left\|D^{2} u\right\|_{S^{0}\left(0, t_{1}\right)}^{2} \leq 1$, we can get

$$
\begin{aligned}
& \left\|D^{2}\left(u_{\omega}-u\right)\right\|_{S^{0}\left(0, t_{1}\right)} \\
& \leq 2 C\left\|D^{2}\left(u_{\omega}(0)-u(0)\right)\right\|_{L^{2}\left(R^{n}\right)} \\
& +2 C\left\|D^{2}\left(u_{\omega}-u\right)\right\|_{S^{0}\left(0, t_{1}\right)}^{(n+4) /(n-4)} \\
& \quad+2\left\|D^{2}\left(u_{\omega}-u\right)\right\|_{S^{0}\left(0, t_{1}\right)}^{8 /(n-4)} \\
& \quad+2\left\|u_{\omega}-u\right\|_{X\left(0, t_{1}\right)}^{(12-n) /(n-4)}+2 C \zeta_{\omega} .
\end{aligned}
$$

Combing (63) and (88), we can obtain

$$
\begin{aligned}
& \left\|D^{2}\left(u_{\omega}-u\right)\right\|_{S^{0}\left(0, t_{1}\right)} \\
& \leq C\left\|D^{2}\left(u_{\omega}(0)-u(0)\right)\right\|_{L^{2}\left(R^{n}\right)}+C\left\|D^{2}\left(u_{\omega}-u\right)\right\|_{S^{0}\left(0, t_{1}\right)}^{(n+4) /(n-4)} \\
& \quad+C\left\|D^{2}\left(u_{\omega}-u\right)\right\|_{S^{0}\left(0, t_{1}\right)}^{8 /(n-4)}+C \zeta_{\omega}+C \xi_{\omega} .
\end{aligned}
$$

Taking $\left\|D^{2}\left(u_{\omega}(0)-u(0)\right)\right\|_{L^{2}\left(R^{n}\right)}, \quad \zeta_{\omega}, \quad \xi_{\omega}$ small such that $2 C\left[2 C\left\|D^{2}\left(u_{\omega}(0)-u(0)\right)\right\|_{L^{2}\left(R^{n}\right)}+2 C \zeta_{\omega}+2 C \xi_{\omega}\right]^{8 /(n-4)}+$ $2 C\left[2 C\left\|D^{2}\left(u_{\omega}(0)-u(0)\right)\right\|_{L^{2}\left(R^{n}\right)}+2 C \zeta_{\omega}+2 C \xi_{\omega}\right]^{(12-n) /(n-4)}<1$, then we can get

$$
\begin{aligned}
& \left\|D^{2}\left(u_{\omega}-u\right)\right\|_{S^{0}\left(0, t_{1}\right)} \\
& \leq 2 C\left\|D^{2}\left(u_{\omega}(0)-u(0)\right)\right\|_{L^{2}\left(R^{n}\right)}+2 C \zeta_{\omega}+2 C \xi_{\omega} .
\end{aligned}
$$

From (83) and (90), we have

$$
\left\|D^{2}\left(u_{\omega}(x, t)-u(x, t)\right)\right\|_{L^{q}\left(0, t_{1} ; L^{r}\left(R^{n}\right)\right)} \longrightarrow 0 \quad \text { as }|\omega| \longrightarrow \infty .
$$

Similarly, let $T_{2}=\sup \left\{t \mid 0<t<l, \| D^{2}\left(u_{\omega}-\right.\right.$ $u) \|_{L^{q}\left(0, t ; L^{r}\left(R^{n}\right)\right)} \rightarrow 0$ as $\left.|\omega| \rightarrow \infty\right\}$. By continuous extension method and contradiction method, we can prove that $T_{2}=l$.

From (53), (67) and (91), the desired result holds.

Lemma 12. Suppose that $n \geq 5$. $(\gamma, \rho)$ is as in Lemma 11. If for any given $M_{1}$, for the case $\|\theta\|_{L^{\infty}(R)} \leq M_{1}$, there exists $\nu\left(M_{1}\right)$ such that $\|W(t) \varphi\|_{L^{\gamma}\left(0, \infty ; L^{\rho}\left(R^{n}\right)\right)} \leq v$, then we have

$$
\begin{gathered}
\left\|D^{2} u_{\omega}\right\|_{L^{\gamma}\left(0, \infty ; L^{\rho}\left(R^{n}\right)\right)} \leq 2 v, \\
\left\|u_{\omega}\right\|_{L^{q}\left(0, \infty ; H^{2, r}\left(R^{n}\right)\right)} \leq C\|\varphi\|_{H^{2}\left(R^{n}\right)},
\end{gathered}
$$

where $(q, r)$ is arbitrary admissible pair and $v$ and $C$ are dependent on $M_{1}$.

Proof. Using (11), Strichartz estimates, and Hölder inequality, noting that $n \geq 5$ (to be sure that $n-4>0$ ), we can get

$$
\begin{aligned}
& \left\|D^{2} u_{\omega}\right\|_{L^{\gamma}\left(0, \infty ; L^{\rho}\left(R^{n}\right)\right)} \\
& \leq\left\|W(t) D^{2} \varphi\right\|_{L^{\gamma}\left(0, \infty ; L^{\rho}\left(R^{n}\right)\right)}+C M_{1}\left\|D^{2} u_{\omega}\right\|_{L^{\gamma}\left(0, \infty ; L^{\rho}\left(R^{n}\right)\right)}^{(n+4) /(n-4)} \\
& \leq \nu+C M_{1}\left\|D^{2} u_{\omega}\right\|_{L^{\gamma}\left(0, \infty ; L^{\rho}\left(R^{n}\right)\right)}^{(n+4) /(n-4)}, \\
& \text { taking } C M_{1} 2^{(n+4) /(n-4)} v^{8 /(n-4)}<1 / 2 \text {, we can get } \\
& \qquad\left\|D^{2} u_{\omega}\right\|_{L^{\gamma}\left(0, \infty ; L^{\rho}\left(R^{n}\right)\right)} \leq 2 \nu .
\end{aligned}
$$

Similarly, we can get

$$
\left\|u_{\omega}\right\|_{L^{\gamma}\left(0, \infty ; H^{2, \rho}\left(R^{n}\right)\right)} \leq C\|\varphi\|_{H^{2}\left(R^{n}\right)},
$$

$$
\begin{aligned}
& \left\|u_{\omega}\right\|_{L^{q}\left(0, \infty ; H^{2, r}\left(R^{n}\right)\right)} \\
& \leq C\|\varphi\|_{H^{2}\left(R^{n}\right)}+C M_{1}\left\|D^{2} u_{\omega}\right\|_{L^{\gamma}\left(0, \infty ; L^{\rho}\left(R^{n}\right)\right)}^{8 /(n-4)} \\
& \quad \times\left\|u_{\omega}\right\|_{L^{\gamma}\left(0, \infty ; L^{\rho}\left(R^{n}\right)\right)} \\
& \leq C\|\varphi\|_{H^{2}\left(R^{n}\right)}+C M_{1}(2 \nu)^{8 /(n-4)} \cdot 2 C\|\varphi\|_{H^{2}\left(R^{n}\right)} \\
& \leq C\|\varphi\|_{H^{2}\left(R^{n}\right)},
\end{aligned}
$$

which completes the proof.

\section{Proofs of Theorems}

Proof of Theorem 3. For any given $0<T<T_{\max }$, suppose that $\|\theta\|_{L^{\infty}\left(R^{n}\right)} \leq M_{1}$; obviously, we have $|I(\theta)| \leq M_{1}$.

For any given $\eta$ (which will be decided later), we divide $[0, T]$ into $\left[t_{i}, t_{i+1}\right], i=1,2, \ldots, J, t_{0}=0, t_{J-1}=T$ such that

$$
\left\|D^{2} u\right\|_{L^{\gamma}\left(t_{i}, t_{i+1} ; L^{\rho}\left(R^{n}\right)\right)} \leq \eta \text {. }
$$


On $\left[t_{0}, t_{1}\right]$, we can get from (12)

$$
\begin{aligned}
& \left\|W(t) D^{2} \varphi\right\|_{L^{\gamma}\left(t_{0}, t_{1} ; L^{\rho}\left(R^{n}\right)\right)} \\
& \leq\left\|D^{2} u\right\|_{L^{\gamma}\left(t_{0}, t_{1} ; L^{\rho}\left(R^{n}\right)\right)}+C M_{1}\left\|D^{2} u\right\|_{L^{\gamma}\left(t_{0}, t_{1} ; L^{\rho}\left(R^{n}\right)\right)}^{8 /(n-4)} \\
& \leq \eta+C M_{1} \eta^{(n+4) /(n-4)} .
\end{aligned}
$$

Let $C M_{1} \eta^{8 /(n-4)}<1$, we have

$$
\left\|W(t) D^{2} \varphi\right\|_{L^{\gamma}\left(t_{0}, t_{1} ; L^{\rho}\left(R^{n}\right)\right)} \leq 2 \eta .
$$

On $\left[t_{1}, t_{2}\right]$, we can get from (12)

$$
\begin{aligned}
& \left\|W(t) D^{2} u\left(t_{1}\right)\right\|_{L^{\gamma}\left(t_{1}, t_{2} ; L^{\rho}\left(R^{n}\right)\right)} \\
& \leq\left\|D^{2} u\right\|_{L^{\gamma}\left(t_{1}, t_{2} ; L^{\rho}\left(R^{n}\right)\right)}+C M_{1}\left\|D^{2} u\right\|_{L^{\gamma}\left(t_{1}, t_{2} ; L^{\prime}\left(R^{n}\right)\right)}^{8 /(n-4)} \\
& \leq \eta+C M_{1} \eta^{(n+4) /(n-4)} .
\end{aligned}
$$

Noting that $C M_{1} \eta^{8 /(n-4)}<1$, we have

$$
\left\|W(t) D^{2} u\left(t_{1}\right)\right\|_{L^{\gamma}\left(t_{1}, t_{2} ; L^{\rho}\left(R^{n}\right)\right)} \leq 2 \eta .
$$

Similarly, on $\left[t_{i}, t_{i+1}\right]$, we can get from (12)

$$
\left\|W(t) D^{2} u\left(t_{i}\right)\right\|_{L^{\gamma}\left(t_{i}, t_{i+1} ; L^{\rho}\left(R^{n}\right)\right)} \leq 2 \eta
$$

Again on $\left[t_{0}, t_{1}\right]$, we can get from (11)

$$
\begin{aligned}
& \left\|D^{2} u_{\omega}\right\|_{L^{\gamma}\left(t_{0}, t_{1} ; L^{\rho}\left(R^{n}\right)\right)} \\
& \leq\left\|W(t) D^{2} \varphi\right\|_{L^{\gamma}\left(t_{0}, t_{1} ; L^{\rho}\left(R^{n}\right)\right)}+C M_{1}\left\|D^{2} u_{\omega}\right\|_{L^{\gamma}\left(t_{0}, t_{1} ; L^{\rho}\left(R^{n}\right)\right)}^{8 /(n-4)} \\
& \leq 2 \eta+C M_{1}\left\|D^{2} u_{\omega}\right\|_{L^{\gamma}\left(t_{0}, t_{1} ; L^{\rho}\left(R^{n}\right)\right)}^{8 /(n-4)} .
\end{aligned}
$$

So we have

$$
\begin{aligned}
& \lim _{\omega \rightarrow \infty}\left\|D^{2} u_{\omega}\right\|_{L^{\gamma}\left(t_{0}, t_{1} ; L^{\rho}\left(R^{n}\right)\right)} \\
& \leq \lim _{\omega \rightarrow \infty}\left(2 \eta+C M_{1}\left\|D^{2} u_{\omega}\right\|_{L^{\gamma}\left(t_{0}, t_{1} ; L^{\rho}\left(R^{n}\right)\right)}^{8 /(n-4)}\right) .
\end{aligned}
$$

Let $2 C M_{1}(4 \eta)^{8 /(n-4)}<1$; we have

$$
\lim _{\omega \rightarrow \infty}\left\|D^{2} u_{\omega}\right\|_{L^{\gamma}\left(t_{0}, t_{1} ; L^{\rho}\left(R^{n}\right)\right)} \leq 4 \eta .
$$

So we know that $\left\|D^{2} u_{\omega}\right\|_{L^{\gamma}\left(t_{0}, t_{1} ; L^{\rho}\left(R^{n}\right)\right)}$ is uniformly bounded. By Lemma 11, we obtain $\lim _{\omega \rightarrow \infty}\left\|D^{2}\left(u_{\omega}\left(t_{1}\right)-u\left(t_{1}\right)\right)\right\|_{L^{2}\left(R^{n}\right)}=$ 0 .
On $\left[t_{1}, t_{2}\right]$, we have

$$
\begin{gathered}
\lim _{\omega \rightarrow \infty}\left\|D^{2} u_{\omega}\right\|_{L^{\gamma}\left(t_{1}, t_{2} ; L^{\rho}\left(R^{n}\right)\right)} \\
\leq \lim _{\omega \rightarrow \infty}\left(\left\|W(t) D^{2} u_{\omega}\left(t_{1}\right)\right\|_{L^{\gamma}\left(t_{1}, t_{2} ; L^{\rho}\left(R^{n}\right)\right)}\right. \\
\left.+C M_{1}\left\|D^{2} u_{\omega}\right\|_{L^{\gamma}\left(t_{1}, t_{2} ; L^{\rho}\left(R^{n}\right)\right)}^{8 /(n-)}\right) \\
\leq \lim _{\omega \rightarrow \infty}\left(\left\|W(t) D^{2}\left(u_{\omega}\left(t_{1}\right)-u\left(t_{1}\right)\right)\right\|_{L^{\gamma}\left(t_{1}, t_{2} ; L^{\rho}\left(R^{n}\right)\right)}\right. \\
+\left\|W(t) D^{2} u\left(t_{1}\right)\right\|_{L^{\gamma}\left(t_{1}, t_{2} ; L^{\rho}\left(R^{n}\right)\right)} \\
\left.+C M_{1}\left\|D^{2} u_{\omega}\right\|_{L^{\gamma}\left(t_{1}, t_{2} ; L^{\rho}\left(R^{n}\right)\right)}^{8 /(n-4)}\right) \\
\leq\left\|W(t) D^{2} u\left(t_{1}\right)\right\|_{L^{\gamma}\left(t_{1}, t_{2} ; L^{\rho}\left(R^{n}\right)\right)} \\
+\lim _{\omega \rightarrow \infty} C M_{1}\left\|D^{2} u_{\omega}\right\|_{L^{\gamma}\left(t_{1}, t_{2} ; L^{\rho}\left(R^{n}\right)\right)}^{8 /(n-4)}
\end{gathered}
$$

Noting that $2 C M_{1}(4 \eta)^{8 /(n-4)}<1$, we have

$$
\lim _{\omega \rightarrow \infty}\left\|D^{2} u_{\omega}\right\|_{L^{\gamma}\left(t_{1}, t_{2} ; L^{\rho}\left(R^{n}\right)\right)} \leq 4 \eta .
$$

Similarly, we have

$$
\lim _{\omega \rightarrow \infty}\left\|D^{2} u_{\omega}\right\|_{L^{\gamma}\left(t_{i}, t_{i+1} ; L^{\rho}\left(R^{n}\right)\right)} \leq 4 \eta .
$$

So we have

$$
\begin{aligned}
& \lim _{\omega \rightarrow \infty}\left\|D^{2} u_{\omega}\right\|_{L^{\gamma}\left(0, T ; L^{\rho}\left(R^{n}\right)\right)} \\
& =\lim _{\omega \rightarrow \infty} \sum_{i=0}^{J-1}\left\|D^{2} u_{\omega}\right\|_{L^{\gamma}\left(t_{i}, t_{i+1} ; L^{\rho}\left(R^{n}\right)\right)} \leq 4 J \eta ;
\end{aligned}
$$

thus by Lemma 11 and Strichartz estimates, Theorem 3 can be obtained.

Proof of Theorem 4. For any $T<+\infty$, we can obtain from Theorem 3

$$
\left\|u_{\omega}(T)-u(T)\right\|_{H^{2}\left(R^{n}\right)} \longrightarrow 0, \quad \text { as }|\omega| \longrightarrow \infty .
$$

For any $\delta>0$, if $T$ is sufficiently large, we immediately get

$$
\left\|D^{2} u\right\|_{L^{\gamma}\left(T, \infty ; L^{\rho}\left(R^{n}\right)\right)} \leq \frac{\delta}{4} .
$$

So using Strichartz estimates and the above inequality, we have

$$
\begin{aligned}
& \left\|W(t) D^{2} u(T)\right\|_{L^{\gamma}\left(T, \infty ; L^{\rho}\left(R^{n}\right)\right)} \\
& \leq\left\|D^{2} u(t)\right\|_{L^{\gamma}\left(T, \infty ; L^{\rho}\left(R^{n}\right)\right)}+C M_{1}\left\|D^{2} u(t)\right\|_{L^{\gamma}\left(T, \infty ; L^{\rho}\left(R^{n}\right)\right)}^{(n+4) /(n-4)} \\
& \leq 2\left\|D^{2} u\right\|_{L^{\gamma}\left(T, \infty ; L^{\rho}\left(R^{n}\right)\right)} \leq \frac{\delta}{2} .
\end{aligned}
$$


If $|\omega|$ is sufficiently large, we can get

$$
\begin{aligned}
& \left\|W(t) D^{2} u_{\omega}(T)\right\|_{L^{\gamma}\left(T, \infty ; L^{\rho}\left(R^{n}\right)\right)} \\
& \leq\left\|W(t)\left(D^{2} u_{\omega}(T)-D^{2} u(T)\right)\right\|_{L^{\gamma}\left(T, \infty ; L^{\rho}\left(R^{n}\right)\right)} \\
& \quad+\left\|W(t) D^{2} u(T)\right\|_{L^{\gamma}\left(T, \infty ; L^{\rho}\left(R^{n}\right)\right)}^{(n+4) /(n-4)} \\
& \leq\left\|D^{2} u_{\omega}(T)-D^{2} u(T)\right\|_{L^{2}\left(R^{n}\right)}+\left\|W(t) D^{2} u(T)\right\|_{L^{\gamma}\left(T, \infty ; L^{\rho}\left(R^{n}\right)\right)}^{(n+4) /(n-4)} \\
& \leq \frac{\delta}{2}+\frac{\delta}{2}=\delta .
\end{aligned}
$$

Using Lemma 12, we have

$$
\begin{gathered}
\left\|D^{2} u_{\omega}\right\|_{L^{\gamma}\left(T, \infty ; L^{\rho}\left(R^{n}\right)\right)} \leq 2 \delta, \\
\left\|u_{\omega}\right\|_{L^{q}\left(T, \infty ; H^{2, r}\left(R^{n}\right)\right)} \leq M_{2},
\end{gathered}
$$

for sufficiently large $|\omega|$.

Similarly, we can get

$$
\begin{gathered}
\left\|D^{2} u\right\|_{L^{\gamma}\left(T, \infty ; L^{\rho}\left(R^{n}\right)\right)} \leq 2 \delta, \\
\|u\|_{L^{q}\left(T, \infty ; H^{2, r}\left(R^{n}\right)\right)} \leq M_{2} .
\end{gathered}
$$

Using Proposition 2 we know $u_{\omega}$ is global.

From Theorem 3, we have

$$
\lim _{|\omega| \rightarrow \infty}\left\|u_{\omega}-u\right\|_{L^{q}\left(0, T ; H^{2, r}\left(R^{n}\right)\right)}=0 .
$$

For the case $t>T$, we have

$$
\begin{aligned}
& u_{\omega}(t)-u(t) \\
& =W(t)\left(u_{\omega}(T)-u(T)\right) \\
& \quad+i \int_{T}^{t} W(t-s) \theta(\omega s)\left|u_{\omega}(s)\right|^{8 /(n-4)} u_{\omega}(s) d s \\
& \quad-i I(\theta) \int_{T}^{t} W(t-s)|u(s)|^{8 /(n-4)} u(s) d s,
\end{aligned}
$$

changing the variable $t$ to $t+T$, we have

$$
\begin{aligned}
u_{\omega}(t+T)-u(t+T) & \\
= & W(t+T)\left(u_{\omega}(T)-u(T)\right) \\
& +i \int_{0}^{t} W(t-s) \theta(\omega(s+T))\left|u_{\omega}(s+T)\right|^{8 /(n-4)} u_{\omega}(s+T) d s \\
& -i I(\theta) \int_{0}^{t} W(t-s)|u(s+T)|^{8 /(n-4)} u(s+T) d s, \\
:= & L_{1}(t)+L_{2}(t)+L_{3}(t), \quad t>0 .
\end{aligned}
$$

Using Strichartz estimates and Hölder inequality, we can get

$$
\begin{aligned}
& \left\|L_{1}(t)\right\|_{L^{q}\left(0, \infty ; H^{2, r}\left(R^{n}\right)\right)} \\
& \quad \leq C\left\|u_{\omega}(T)-u(T)\right\|_{H^{2}\left(R^{n}\right)}, \\
& \left\|L_{2}(t)\right\|_{L^{q}\left(0, \infty ; H^{2, r}\left(R^{n}\right)\right)} \\
& \quad \leq C\left\|D^{2} u_{\omega}\right\|_{L^{\gamma}\left(T, \infty ; L^{\rho}\left(R^{n}\right)\right)}^{8 /(n-4)}\left\|u_{\omega}\right\|_{L^{\gamma}\left(T, \infty ; L^{\rho}\left(R^{n}\right)\right)} \\
& \quad \leq C(2 \delta)^{8 /(n-4)} M_{2}, \\
& \left\|L_{3}(t)\right\|_{L^{q}\left(0, \infty ; H^{2, r}\left(R^{n}\right)\right)} \\
& \quad \leq C\left\|D^{2} u\right\|_{L^{\gamma}\left(T, \infty ; L^{\rho}\left(R^{n}\right)\right)}^{8 /(n-4)}\|u\|_{L^{\gamma}\left(T, \infty ; L^{\rho}\left(R^{n}\right)\right)} \\
& \quad \leq C(2 \delta)^{8 /(n-4)} M_{2} .
\end{aligned}
$$

So we have

$$
\begin{aligned}
\lim _{|\omega| \rightarrow \infty}\left\|u_{\omega}-u\right\|_{L^{q}\left(T, \infty ; H^{2, r}\left(R^{n}\right)\right)} \leq 2 C(2 \delta)^{8 /(n-4)} M_{2} \\
\lim _{|\omega| \rightarrow \infty}\left\|u_{\omega}-u\right\|_{L^{q}\left(0, \infty ; H^{2, r}\left(R^{n}\right)\right)} \\
\leq \lim _{|\omega| \rightarrow \infty}\left\|u_{\omega}-u\right\|_{L^{q}\left(0, T ; H^{2, r}\left(R^{n}\right)\right)} \\
\quad+\lim _{|\omega| \rightarrow \infty}\left\|u_{\omega}-u\right\|_{L^{q}\left(T, \infty ; H^{2, r}\left(R^{n}\right)\right)} \\
\leq 2 C(2 \delta)^{8 /(n-4)} M_{2} .
\end{aligned}
$$

Let $\delta \rightarrow 0$; we can get

$$
\lim _{|\omega| \rightarrow \infty}\left\|u_{\omega}-u\right\|_{L^{q}\left(0, \infty ; H^{2, r}\left(R^{n}\right)\right)}=0,
$$

which completes the proof of Theorem 4.

\section{Conflict of Interests}

The authors declare that there is no conflict of interests regarding the publication of this paper.

\section{Acknowledgments}

This work is supported by Natural Science of Shanxi province (no. 2013011003-2, no. 2013011002-2, and no. 2010011001-1), Natural Science Foundation of China (no. 11071149), and Research Project Supported by Shanxi Scholarship Council of China (no. 2011-011).

\section{References}

[1] T. Cazenave, Semilinear Schrodinger Equations, American Mathematical Society, 2003.

[2] V. I. Karpman, "Stabilization of soliton instabilities by higherorder dispersion: fourth-order nonlinear Schrödinger-type equations," Physical Review E-Statistical Physics, Plasmas, Fluids, and Related Interdisciplinary Topics, vol. 53, no. 2, pp. R1336R1339, 1996. 
[3] V. I. Karpman and A. G. Shagalov, "Stability of solitons described by nonlinear Schrödinger-type equations with higher-order dispersion," Physica D, vol. 144, no. 1-2, pp. 194-210, 2000.

[4] C. Miao, G. Xu, and L. Zhao, "Global well-posedness and scattering for the focusing energy-critical nonlinear Schrödinger equations of fourth order in the radial case," Journal of Differential Equations, vol. 246, no. 9, pp. 3715-3749, 2009.

[5] C. Miao, G. Xu, and L. Zhao, "Global well-posedness and scattering for the defocusing energy-critical nonlinear Schrödinger equations of fourth order in dimensions $d \geq 9$," Journal of Differential Equations, vol. 251, no. 12, pp. 3381-3402, 2011.

[6] B. Pausader, "Global well-posedness for energy critical fourthorder Schrödinger equations in the radial case," Dynamics of Partial Differential Equations, vol. 4, no. 3, pp. 197-225, 2007.

[7] B. Pausader, "The cubic fourth-order Schrodinger equation," Journal of Functional Analysis, vol. 256, no. 8, pp. 2473-2517, 2009.

[8] J. Zhang and J. Zheng, "Energy critical fourth-order Schrödinger equations with subcritical perturbations," Nonlinear Analysis: Theory, Methods \& Applications, vol. 73, no. 4, pp. 1004-1014, 2010.

[9] D. Fang and Z. Han, "A Schrödinger equation with timeoscillating critical nonlinearity," Nonlinear Analysis: Theory, Methods \& Applications, vol. 74, no. 14, pp. 4698-4708, 2011.

[10] S. B. Cui and C. H. Guo, "Well-posedness of higher-order nonlinear Schrödinger equations in SOBolev spaces $H^{s} \mathscr{R}^{n}$ and applications," Nonlinear Analysis: Theory, Methods \& Applications, vol. 67, no. 3, pp. 687-707, 2007.

[11] R. A. Adams and J. J. Fourier, Sobolev Spaces, Springer, New York, NY, USA, 2009.

[12] J. Bergh and J. Lofstrom, Interpolation Spaces, Springer, New York, NY, USA, 1976.

[13] R. Killip and M. Visan, "Nonlinear Schrödinger equation at critical regularity," Clay Mathematics Proceedings, vol. 17, pp. 1113, 2013.

[14] T. Cazenave and M. Scialom, "A Schrödinger equation with time-oscillating nonlinearity," Revista Matemática Complutense, vol. 23, no. 2, pp. 321-339, 2010. 


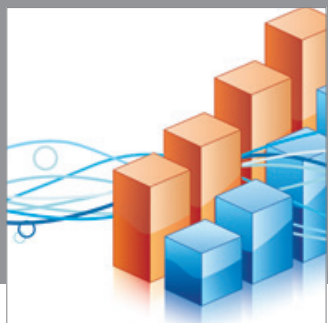

Advances in

Operations Research

mansans

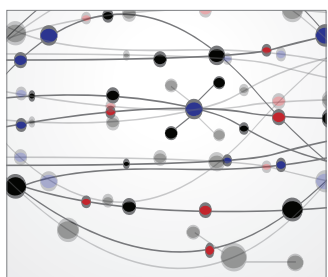

The Scientific World Journal
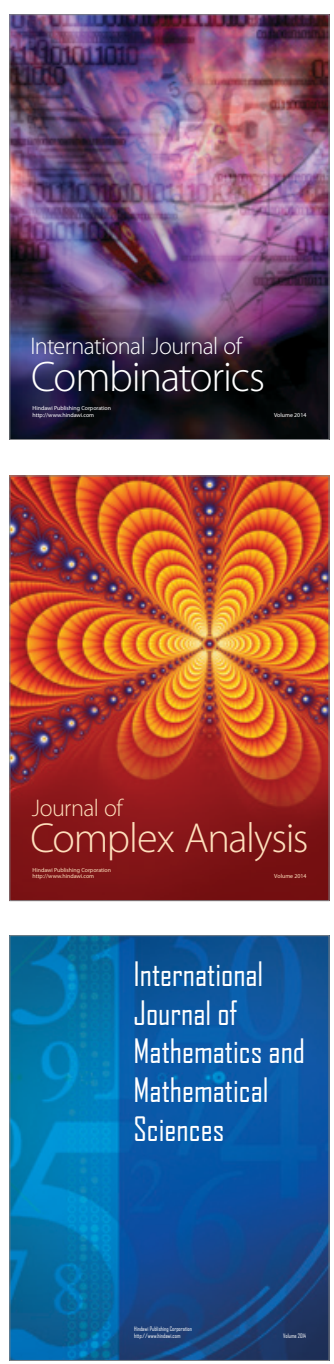
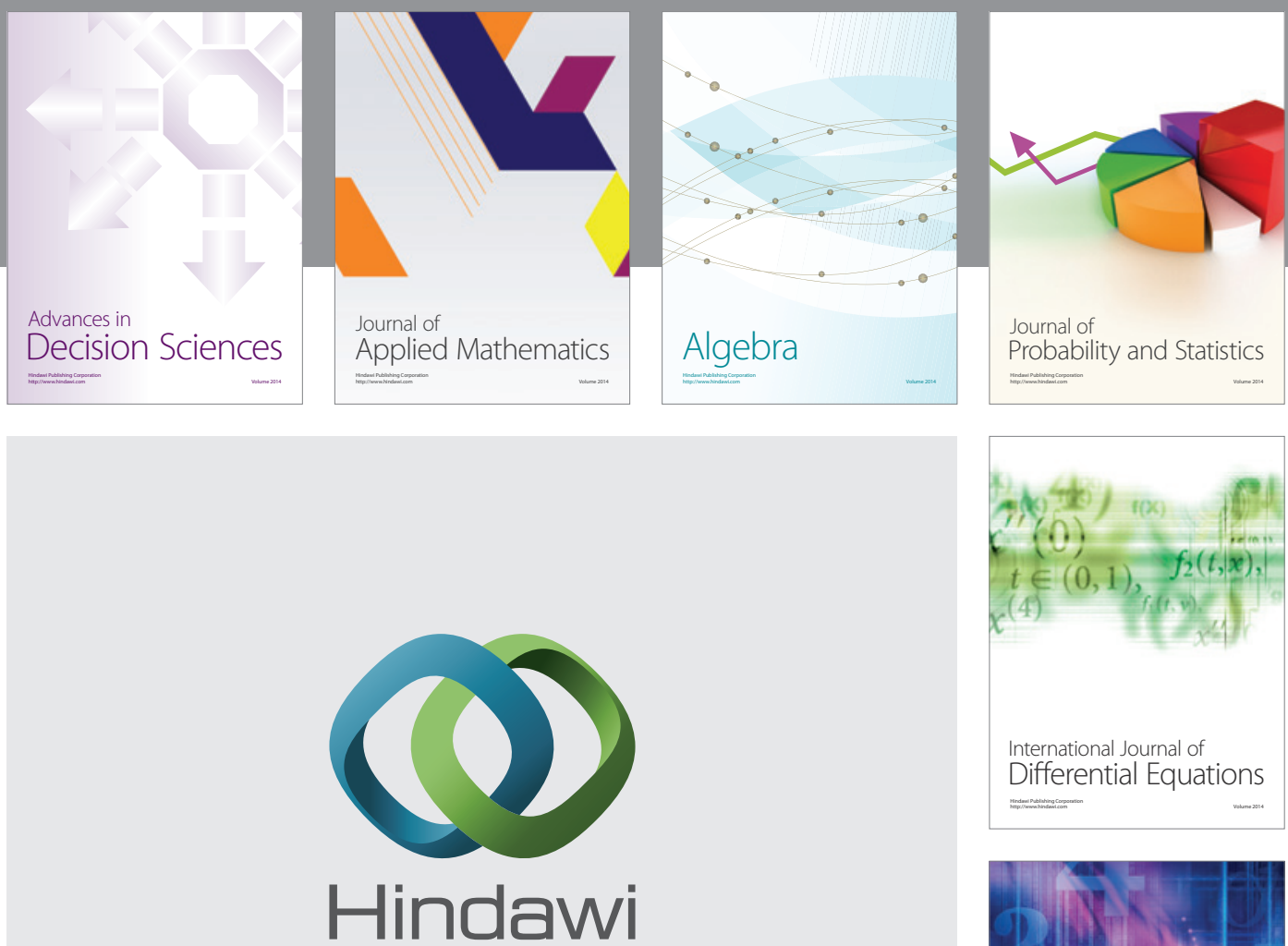

Submit your manuscripts at http://www.hindawi.com
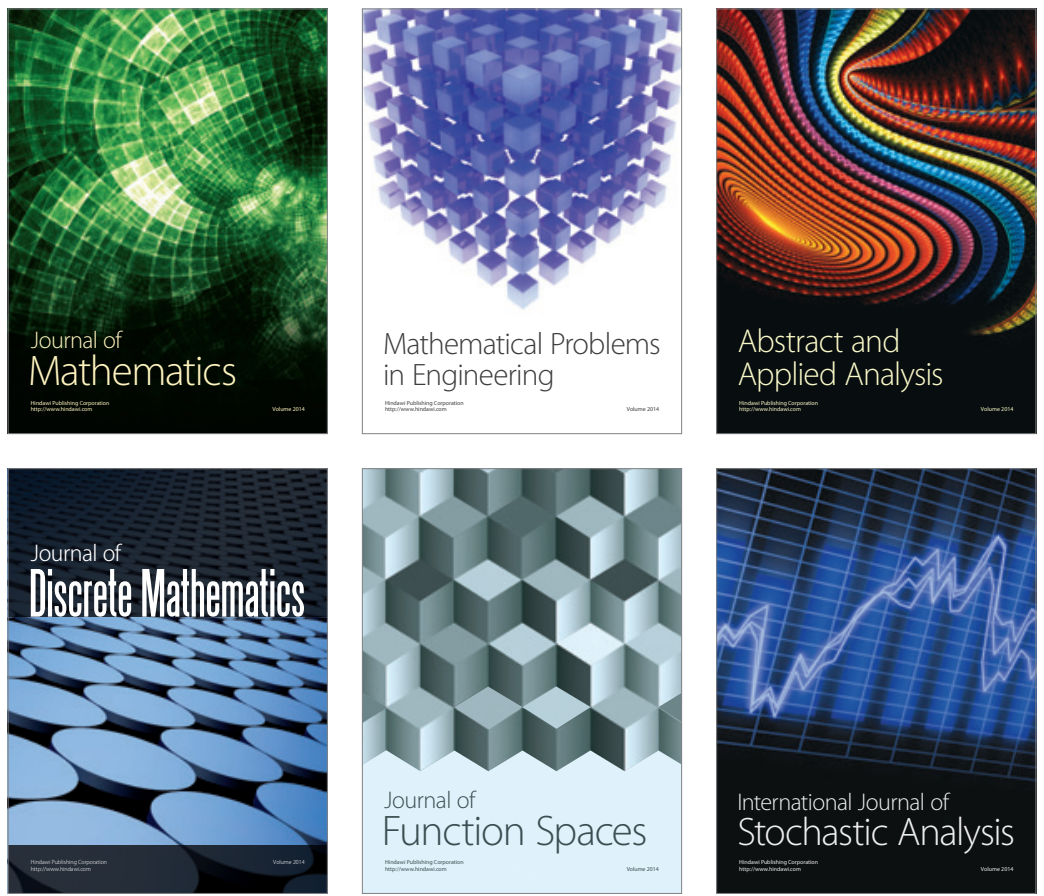

Journal of

Function Spaces

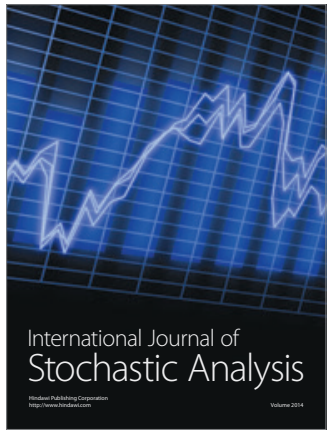

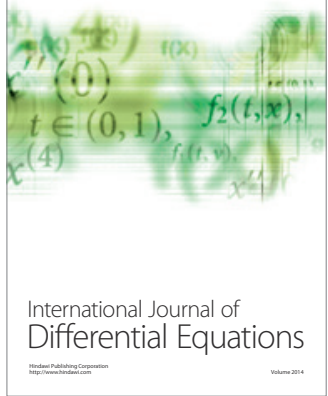
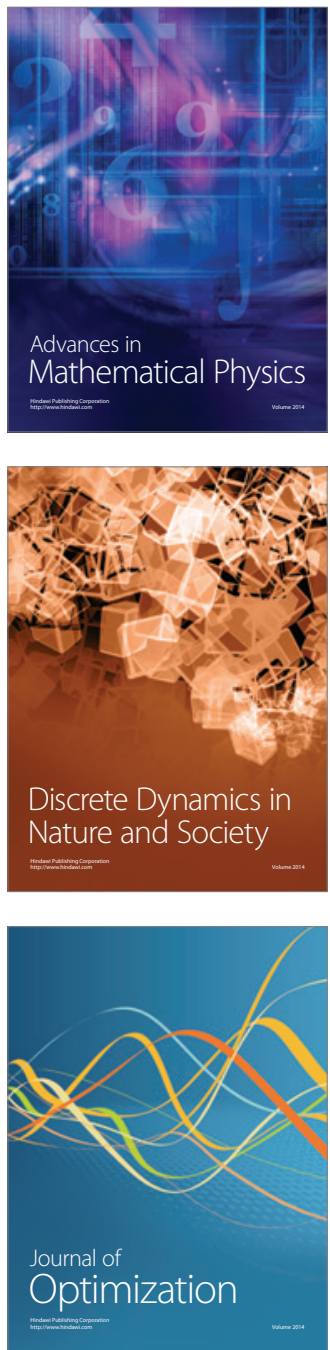